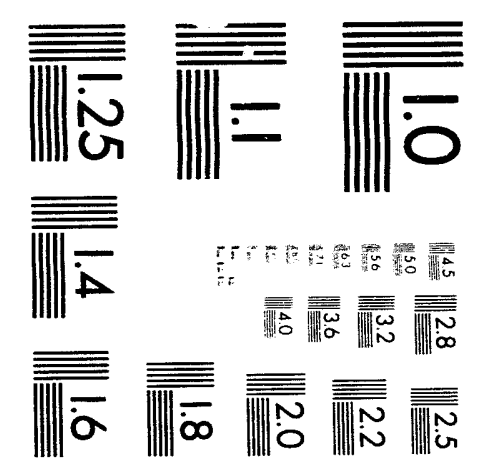



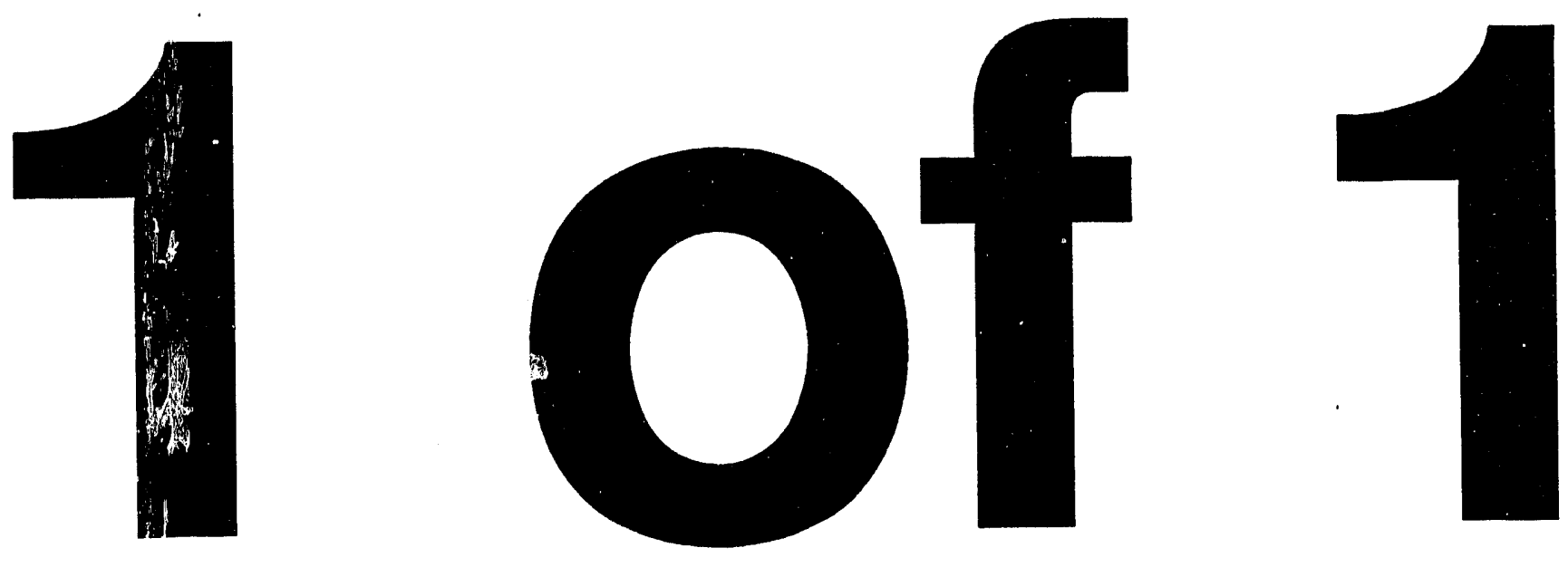


\title{
Nonlinear Ion-Mixing-Mode Particle Transport in the Dissipative Trapped Electron Regime
}

\author{
A. S. Ware and P. W. Terry \\ Department of Physics \\ University of Wisconsin-Madison \\ Madison, Wisconsin 53706
}

\begin{abstract}
The nonlinear particle transport arising from the convection of nonadiabatic electron density by ion temperature gradient driven turbulence is examined for trapped electron collisionality regimes. The renormalized dissipative nonadiabatic trapped electron phase space density response is derived and used to calculate the nonlinear particle flux along with an ansatz for the turbulently broadened frequency spectrum. In the lower temperature end of this regime, trapped electrons are collisional and all components of the quasilinear particle flux are outward (i.e., in the direction of the gradients). Nonlinear effects can alter the phase between the nonadiabatic trapped electron phase space density and the electrostatic potential, producing inward components in the particle flux. Specifically, both turbulent shifting of the peak of the frequency spectrum and nonlinear source terms in the trapped electron response can give rise to inward components. However, in the dissipative regime these terms are small and the trapped electron response remains dominantly laminar. When the trapped electrons are collisionless, there is a temperature threshold above which the electron temperature gradient driven component of the quasilinear particle flux changes sign and becomes inward. For finite amplitude turbulence, however, turbulent broadening of both the electron collisional resonance and the frequency spectrum removes this threshold, and the temperature gradient driven component remains outward.
\end{abstract}




\section{INTRODUCTION}

It has been established that turbulent fluctuations are capable of transporting heat and particles at the anomalous rates observed in experiment. In many cases, these rates greatly exceed collisional transport rates. While both particles and heat are transported as a result of fluctuations, the loss of heat has generally been regarded as the more serious concern. This view is supported by the fact that the convective component of the heat flux (representing heat loss due to particle transport) is often small compared to the conductive heat flux in the core of the plasma. In recent years, interesting correlations between the density profile and thermal confinement improvement. ${ }^{1-3}$ have been increasingly evident. These observations suggest that particle transport (which governs the density profile along with particle sources) has important, if indirect, links to global confinement. Accordingly, a number of recent experimental studies of core transport have specifically examined particle losses in detail, ${ }^{4-6}$ thus providing another point of comparison between experiment and theory.

An interesting question raised by studies of particle transport is the possible existence of a particle pinch. Typically modelled as a negative (inward) particle convection which accompanies outward diffusive transport, ${ }^{7}$ the pinch is necessary in order to maintain peaked density profiles in a steady state configuration against diffusive losses assuming the particle source is negligible in the core. This robust, albeit simple, rationale for the pinch is further supported by the results of perturbative transport analyses. Using a linear diffusion/convection model $\left(\Gamma=-D \partial n / \partial r-v_{0} n r / a\right.$, where $D$ and $v_{0}$ can vary spatially but are independent of $n$ and $\partial n / \partial r$ ) for the particle flux arising in a discharge with a modulated gas feed, it was found that a strong inward convection velocity of tens of meters per second was required to model the discharges. ${ }^{5}$ A more recent study in TFTR inas concluded that the particle pinch required for the plasma to respond to an oscillating gas puff is markedly smaller. In this study, the flux was modeled as a general nonlinear functional 
of the density, density gradient, temperature, and temperature gradient, with its precise form determined by a fit to the data. This general model removes the artificial constraint that the flux take the diffusion/convection form with linear coefficients and is therefore more consistent with the reasonable view that transport coefficients are functions of the turbulence level, which in turn must depend on the driving gradients. While this result indicates that the flux model can make a large difference in the inferred magnitude of the inward pinch, it should not be interpreted as indication that the particle pinch is small in steady state conditions. Steady state transport has temperature gradient driven components whereas the gas puff perturbation in TFTR produced only a very weak perturbation of the temperature gradient. One long-standing anomalous pinch mechanism is, in fact, produced by the temperature gradient. ${ }^{7-10}$ The TFTR data therefore, leaves open the question of the existence of a pinch in steady state discharges.

One mechanism capable of producing an inward particle flux in specific electron sollisionality regimes is the ion mixing mode ${ }^{7-10}$ an effect arising from the convection of nonadiabatic electron density fluctuations by ion temperature gradient driven turbulence. The dissipative nonadiabatic electron density response governs the ambipolar particle transport (by virtue of its phase shift relative to the potential) but is assumed to produce only minor changes in the ion driving mechanism which excites the turbulence. The ion mixing mode produces a fux component proportional to the density gradient (nominally diffusive) which is outward, and an off-diagonal temperature gradient driven component which can either be inward or outward. It is instructive to note that the net flux produced by $\mathrm{E} \times \mathrm{B}$ convection of fluctuations in the nonadiabatic electron density is identical in form to the particle flux driven by electron drift wave turbulence, provided the fluctuation level is left unspecified. Indeed, if the fluctuation level is supplied by experimental measurement (as opposed to using a level obtained from model dependent saturation conditions), the flux represents a generic particle transport description for quasineutral, low frequency electrostatic fluctuations. In the case of electron drift waves, instability is governed by the same correlation 
which describes the turbulent convection responsible for particle transport. When the sign of the correlation is such as to produce an inward flux. drift waves are stable and finite amplitude turbulence is not possible. In contrast, ion temperature gradient driven turbulence is driven by ion processes. Hence, the correlation describing convection of electron density is of negligible consequence to the driving mechanism provided $\omega_{*} / k_{\|} v_{i}>\delta_{e}$ where $\omega_{*}$ is the diamagnetic frequency, $v_{i}$ the ion thermal velocity and $\delta_{e}$ represents the nonadiabatic electron density response: $\operatorname{Im}\left\langle\tilde{\mathrm{n}} / \mathrm{n}_{0} \cdot e \bar{\phi} / \mathrm{T}_{e}\right\rangle=\delta_{e}\left\langle\left|e \bar{\phi} / \mathrm{T}_{e}\right|^{2}\right\rangle$.

Previous calculations of the ion mixing mode particle flux have examined all relevant electron collisionality regimes, but have been restricted to a quasilinear treatment. For regimes with sufficient collisionality to prevent electron trapping $\left(\nu_{e f f}=\nu_{e} / \epsilon>\omega_{b}\right.$, where $\nu_{e}$ is the electron collision frequency, $\epsilon$ is the inverse aspect ratio, and $\omega_{b}$ the electron bounce frequency), the temperature gradient driven component is inward and exceeds the outward density gradient driven component. ${ }^{8,9}$ This net inward flux requires sufficient collisionality to prevent trapping, and thus operates in colder edge regions, enabling source particles to penetrate into the plasma. In the hotter dissipative trapped electron regime where the collisionality is sufficiently low to allow trapping of electrons $\left(\nu_{e f f}<\omega_{b}\right)$, but not so low that it becomes smaller that the mode frequency $\left(\nu_{e}>\omega\right)$, the flux is wholly outward. ${ }^{7.9}$ For the still hotter collisionless trapped electron regime $\left(\nu_{e}<\omega_{b}, \nu_{e}<\omega\right)$, the temperature gradient driven component becomes inward above a critical temperature somewhat higher than the temperature for which $\nu_{e}<\omega{ }^{7,10}$ This inward component is sufficient to balance the outward diffusive losses (from the density gradient driven component) and enable a stationary density profile. Unfortunately, there is no such possibility with quasilinear estimates in the dissipative trapped electron regime. In moderately sized ohmically heated devices such as TEXT, this regime occupies a sizable fraction of the core confinement zone, and is likely to have a small particle source at best.

The existence of other pinch mechanisms has been noted. ${ }^{11-13}$ In the edge, ionization of neutral particles drives a drift wave whose particle flux. like that of the ordinary linearly 
stable collisional drift wave, is inward. ${ }^{11}$ With this mode, the ionization source produces instability even though the flux is inward. This mechanism facilitates the penetration of source particles, but is not operative beyond the ionization or source region. Another mechanism, ${ }^{13}$ based on asymmetric potential structure, has been proposed but is difficult to assess as little is known of experimental asymmetries. If these asymmetries arise from boundary conditions through, for example, a limiter, ${ }^{14}$ this mechanism is also restricted to the edge. An interesting study of basic nonlinear drift wave dynamics has uncovered components of the particle flux which are inward and are directly related to the dynamical evolution of the correlation of fluctuating density and potential. ${ }^{12}$ The evolution of this correlation is typically ignored in drift wave models and is not captured by quasilinear transport estimates of mixing mode fluxes. This issue is directly related to mechanisms which will be examined in the present work and discussed later.

In the present paper, the particle flux associated with low frequency, quasineutral, electrostatic density and potential fluctuations will be extended beyond previous quasilinear calculations to include a variety of nonlinear effects. The principal focus is mixing mode transport in the dissipative trapped electron collisionality regime, where at present no viable pinch mechanism has been identified. However, as already noted, if the fluctuation level $\left|e \tilde{\phi} / T_{e}\right|^{2}$ is left unspecified, this analysis is generally applicable to electrostatic microturbulence. This calculation thus represents a more accurate description of particle transport for comparison with experiment than has heretofore been available. ${ }^{6}$ The nonlinear effects incorporated in this analysis derive primarily, but not entirely, from a renormalized electron phase space density response, and are therefore consistent with a strong turbulence approach. However, because the turbulent mixing rates which enter the particle propagators are amplitude dependent, weak turbulence limits, i.e. quasilinear theory, are also recovered. The nonlinear processes which are treated in the electron distribution function evolution are wave-electron resonance broadening, the nonlinear shift in the wave-electron resonance frequency (which appears as the imaginary part of the complex 
wave-electron resonance broadening), and the renormalization of the source or potential frequently referred to as the $\beta$ term. ${ }^{14}$ The latter is proportional to the correlation of the phase space density with the potential and as such introduces elements associated with the evolution of the correlation of density with potential. In addition to the nonlinearities of the renormalized electron phase space density, a turbulently broadened frequency spectrum is assumed. Modelled as a Lorentzian to facilitate integration over frequency, the spectrum includes a linewidth (frequency spectrum width for a fixed wavenumber $\mathrm{k}$ ) produced by the turbulent eddy damping which balances incoherent emission in the steady state. ${ }^{15} \mathrm{~A}$ nonlinear shift of the peak away from the linear frequency $\omega_{k}$ is also retained. The shift is an observed feature of mode-coupling simulations ${ }^{16}$ and can arise as a fluctuation induced modification of diamagnetic drift effects. ${ }^{17}$

The results of the present calculation indicate that the nonlinear effects do give rise to inward components of the particle flux in the dissipative trapped electron regime. These terms derive primarily from the frequency spectrum shift and the $\beta$ terms. The effect of the frequency shift is readily understood heuristically from the factor $\left(\omega-\omega_{* e}\right)$ in the flux. Since the flux includes a spectrum weighted integral over frequency of the electron density response, the sensitivity to the shifts is not surprising. Nor is the general insensitivity to the broadening surprising. given that in absence of the shifts, the broadening terms tend to produce both higher and lower frequencies, with offsetting effects on the flux. The role of the $\beta$ terms in producing inward flux components is less easy to describe heuristically. However, the fact that the $\beta$ terms are proportional to a phase space density - potential correlation, while the same correlation (integrated over velocity) governs the flux, suggests that a strong effect of one on the other is plausible.

The nonlinear flux calculated herein is a highly complicated function due to the interplay of the various nonlinearities, their dependence on fluctuation amplitude, and the fact that the nonlinearities are defined recursively in the strong turbulence limit. Quantitative evaluation of the resulting terms is difficult, particularly for strong turbulence. 
and will be addressed elsewhere. However, it is possible to make the following qualitative statements regarding the flux. The nonlinear terms neglected in quasilinear analyses can be important and make significant differences at fluctuation levels consistent with crude mixing length arguments, i.e., $\left|e \bar{\phi} / \mathrm{T}_{e}\right| \sim \Delta / \mathrm{L}_{n}$, where $\Delta$ is a radial correlation length and $L_{n}$ is the density gradient scale length. If the fluctuations responsible for the transport saturate via a classical weak turbulence mechanism, ${ }^{18}$ the additional factor of $\gamma / \omega$ renders the contribution of the nonlinear terms to the flux small. For ion temperature gradient driven turbulence (ion mixing mode), the large correction factor associated with the effect of broader fluctuation structure ${ }^{19}$ enhances the fluctuation level and significant nonlinear modifications occur. At vanishingly small fluctuation levels, the nonlinear particle flux approaches the quasilinear prediction. As the fluctuation level increases to finite amplitude (and, hence the linewidth of the frequency spectrum as well), the nonlinear flux can deviate greatly from the quasilinear flux. In the strongly dissipative regime, the electron response is still dominantly laminar and the nonlinear effects tend to decrease the magnitude of the outwardly directed flux below the quasilinear level. In the moderately and weakly dissipative regimes, the nonlinear effects remove the pinch associated with the quasilinear $\nabla T_{e}$ term. In the collisionless limit, this can change the sign of the overall particle flux; from inward in the quasilinear aialysis to outward in the nonlinear analysis.

The remainder of this paper is organized as follows. Section II contains a derivation of the renormalized trapped electron phase space density response. In Section III, the nonlinear particle flux is calculated using the renormalized trapped electron response and an ansatz of a turbulently broadened frequency spectrum. The results of this work are discussed in Section IV. 


\section{RENORMALIZED TRAPPED ELECTRON EQUATION}

In this section, we derive and examine a renormalized trapped electron phase space density response in both the dissipative and collisionless regimes. Consistent with previous theories of ion-temperature-gradient-driven turbulence. ${ }^{0.19,20}$ we consider a radially inhomogeneous slab of plasma with the magnetic field given by $\mathbf{B}=B_{0}\left[\hat{z}+\hat{y}\left(x / L_{s}\right)\right]$, where $L_{s}^{-1}=B_{0}^{-1} \partial B_{y} / \partial x$.

The particle flux is given by the $\mathbf{E} \times \mathbf{B}$ convection of the nonadiabatic density

$$
\begin{aligned}
& \Gamma_{e}=\left\langle\bar{v}_{r}, \tilde{n}_{e}\right\rangle \\
& =\sum_{\substack{k \\
\omega}} \frac{c}{B} i i_{y}\left\langle\frac{e \bar{\phi}-k}{T_{e}} n_{e j}{ }_{w}\right\rangle \\
& =-n_{e} c_{s} \sum_{\substack{k \\
\omega}} \rho_{s} k_{y} \operatorname{Im}\left\langle\frac{e \bar{\phi}-k}{T_{e}} \int d^{3} v \tilde{h}_{w}\right\rangle \text {. }
\end{aligned}
$$

where $c_{s}=\sqrt{T_{e} / m_{i}}, \rho_{s}=c_{s} / \Omega_{i}$, and the density has been separated into an adiabatic response and an integral over a nonadiabatic phase space density response $\bar{n}_{e^{k}} / n_{\varepsilon}=$ $e \tilde{\phi}_{w} / T_{e}+\int d^{3} v \bar{h}_{k}$. The angular brackets in Eq. (1), $\langle\cdots\rangle$, represent an ensemble average or, effectively, an average over the radial coordinate.

The nonadiabatic trapped electron density satisfies a nonlinear gyrokinetic equation, given by

$$
\begin{aligned}
\left(\omega-\omega_{d e} \frac{v^{2}}{v_{e}^{2}}+\frac{\nu_{e}}{\epsilon} \frac{v_{e}^{3}}{v^{3}}\right) \tilde{h}_{\omega}+N_{\omega} \\
=\left\{\omega-\omega_{* e}\left[1+\eta_{e}\left(\frac{v^{2}}{v_{e}^{2}}-\frac{3}{2}\right)\right]\right\} \frac{e \bar{\phi}_{\omega}}{T_{e}} F_{\text {max }},
\end{aligned}
$$

where $\omega_{d e}=\epsilon_{n} \omega_{* e}, \omega_{* e}=\left(c T_{e} / e B\right) k_{y} / L_{n}, \epsilon_{n}=L_{n} / R, \eta_{e}=L_{n} / L_{T}=d \ln T_{e} / d \ln n_{e}$, $\epsilon=r / R$, and $v_{e}=\sqrt{T_{e} / m_{e}}$. The Fourier transformed $\mathbf{E} \times \mathbf{B}$ nonlinearity is given by

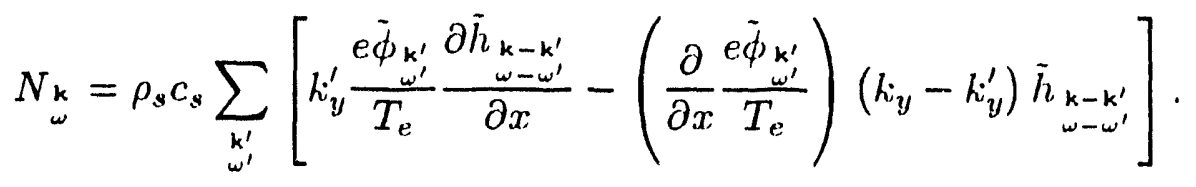


Quasilinear analysis of the particle flux ignores the nonlinearity and assumes a delta function for the frequency spectrum, i.e. $\left\langle\tilde{\phi}^{2}\right\rangle_{k}=\pi \delta\left(\omega-\omega_{k}\right)\left\langle\tilde{\phi}^{2}\right\rangle_{k}$. While this may be sufficient in a weak turbulence or strongly dissipative regime $\left(\nu_{\text {eff }} \gg \omega_{* e}, \omega\right)$, it is clearly not appropriate in the moderately dissipative or collisionless regimes in the presence of moderate or strong turbulence. In order to improve upon previous theory, we use a statistical closure method to iteratively renormalize Eq. (2).

In the iterative procedure, $\tilde{h}_{k-k^{\prime}}$ is replaced in Eq. (2) with the beat driven fluctuation $\tilde{h}_{\substack{k-k^{\prime} \\ w-w^{\prime}}}^{(2)}$. The beat driven phase space density fluctuation satisfies the equation

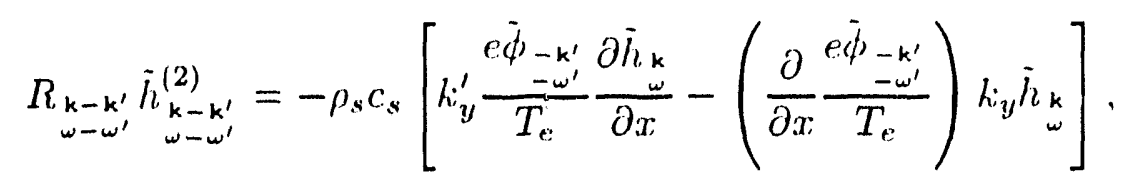

where $R_{\substack{k-k^{\prime} \\ \omega-\omega^{\prime}}}=\omega-\omega^{\prime}-\epsilon_{n}\left(\omega_{* e}-\omega_{* e}^{\prime}\right) \frac{v^{2}}{v_{e}^{2}}+i \frac{\nu_{c}}{\epsilon} \frac{v_{c}^{3}}{v^{3}}+i \nu_{\substack{k-k^{\prime} \\ \omega-w^{\prime}}}$ is the trapped electron propagator and $\nu_{k-k^{\prime}}$ represents the turbulent collisional resonance broadening. The beat driven potential, $\dot{\phi}_{\substack{k-k^{\prime} \\ w-w^{\prime}}}^{(2)}$, is ignored due to the difficulties in solving the integro-differential equation necessary for including it and the narrow radial extent of the region in which the $\bar{\phi}_{k-k^{\prime}}^{(2)}$ perturbation is significant. ${ }^{21}$

Using the beat driven fluctuation, the renormalized trapped electron equation is given by

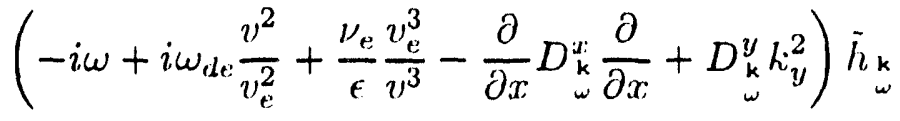

$$
\begin{aligned}
& =i\left\{\omega-\omega_{* e}\left[1+\eta_{e}\left(\frac{v^{2}}{v_{e}^{2}}-\frac{3}{2}\right)\right]+\frac{\partial}{\partial x} \beta_{k}^{x} \frac{\partial}{\partial x}-\beta_{\substack{y \\
\omega}}^{2}\right\} \frac{e \bar{\phi}_{k}}{T_{e}} F_{\text {max }}
\end{aligned}
$$

where the renormalized coefficients are

$$
\begin{aligned}
& D_{\substack{k \\
w}}^{x}=\rho_{s}^{2} c_{s}^{2} \sum_{\substack{k^{\prime} \\
w^{\prime}}} R_{\substack{k-k^{\prime} \\
\omega-w^{\prime}}}^{-1} k_{y}^{\prime 2}\left\langle\left(\frac{e \tilde{\phi}}{T_{e}}\right)^{2}\right\rangle_{\substack{k^{\prime} \\
\omega^{\prime}}}, \\
& D_{\substack{k \\
\omega}}^{y}=\rho_{s}^{2} c_{s}^{2} \sum_{\substack{k^{\prime} \\
\omega^{\prime}}} R_{\substack{k-k^{\prime} \\
\omega-w^{\prime}}}^{-1}\left(\left(\frac{\partial}{\partial x} \frac{e \tilde{\phi}}{T_{e}}\right)^{2}\right\rangle_{\substack{k^{\prime} \\
\omega^{\prime}}},
\end{aligned}
$$




$$
\begin{aligned}
& \beta_{\substack{k \\
\omega}}^{x}=\rho_{s}^{2} c_{s}^{2} \sum_{\substack{k^{\prime} \\
\omega^{\prime}}} i R_{\substack{k-k^{\prime} \\
\omega-\omega^{\prime}}}^{-1} k_{y}^{\prime 2}\left\langle h \frac{e \bar{\phi}}{T_{e}}\right\rangle_{\substack{k^{\prime} \\
\omega^{\prime}}}\left(F_{\text {max }}\right)^{-1}, \\
& \beta_{\substack{k \\
\omega}}^{y}=\rho_{s}^{2} c_{s}^{2} \sum_{\substack{k^{\prime} \\
\omega^{\prime}}} i R_{\substack{k-k^{\prime} \\
\omega-\omega^{\prime}}}^{-1}\left\langle\frac{\partial h}{\partial x} \frac{\partial}{\partial x} \frac{e \bar{\phi}}{T_{e}}\right\rangle_{\substack{k^{\prime} \\
\omega^{\prime}}}\left(F_{\text {maxis }}\right)^{-1} .
\end{aligned}
$$

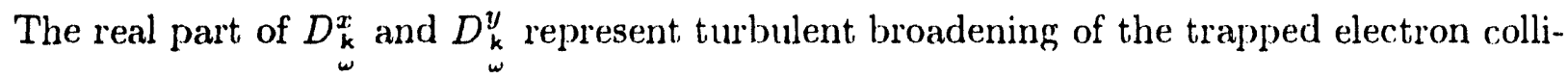
sional resonance while the imaginary part of these terms represents a shift in the peak of the resonance. $\beta_{k}^{x}$ and $\beta_{k}^{y}$ are the renormalized source terms ${ }^{14}$ which allow nonlinear access to the free energy source and are energy conserving terms (when summed over $k$ ). These terms are related to the cross correlation of the phase space density with the potential. In this one-point analysis, we will recursively define these terms using the renormalized trapped electron equation to determine the phase between $\bar{h}_{\substack{\boldsymbol{k}^{\prime} \\ w^{\prime}}}$ and $\frac{e \bar{\phi}_{\boldsymbol{k}}^{\prime}}{{\overline{w^{\prime}}}_{e}}$. A more complete two-point analysis would involve an equation for the evolution of the cross correlation. ${ }^{13}$ however, that is beyond the scope of this work. The source terms are thus

$$
\begin{aligned}
& \beta_{\substack{k \\
\omega}}^{x}=\rho_{s}^{2} c_{s}^{2} \sum_{\substack{k^{\prime} \\
\omega^{\prime}}} R_{\substack{k-k^{\prime} \\
\omega-\omega_{0}^{\prime}}}^{-1} R_{\substack{-\boldsymbol{k}^{\prime} \\
-\omega_{0}^{\prime}}}^{-1} k_{y}^{\prime 2}\left\langle\left(\frac{e \bar{\phi}}{T_{e}}\right)^{2}\right\rangle_{\substack{k^{\prime} \\
\omega^{\prime}}}\left\{\omega^{\prime}-\omega_{* e}^{\prime}\left[1+\eta_{e}\left(\frac{v^{2}}{v_{e}^{2}}-\frac{3}{2}\right)\right]-\beta^{\prime}\right\} .
\end{aligned}
$$

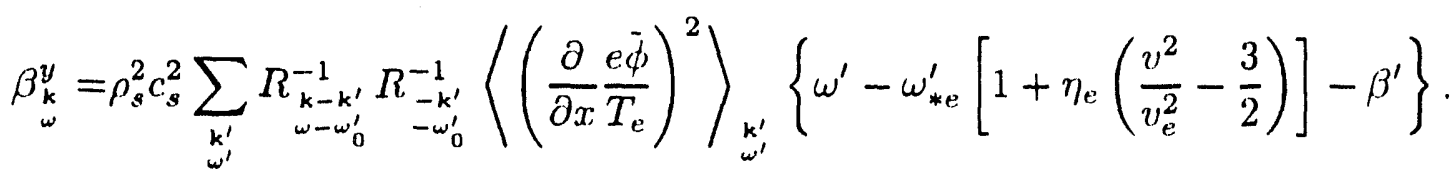

To further improve upon quasilinear theory, an ansatz of a Lorentzian frequency spectrum is assumed:

$$
\left\langle\left(\frac{e \tilde{\phi}}{T_{e}}\right)^{2}\right\rangle_{\substack{\omega \\ j}}=\frac{\Delta \omega_{k} / \pi}{\left(\omega-\omega_{k}^{N L}\right)^{2}+\left(\Delta \omega_{k}\right)^{2}}\left\langle\left(\frac{e \tilde{\phi}}{T_{e}}\right)^{2}\right\rangle_{\mathbf{k}}
$$

where $\Delta \omega_{k}$ represents the finite linewidth of the frequency spectrum induced by incoherent emission $^{9,22}$ and $\omega_{k}^{N L}=\omega_{k}+\delta \omega_{k}$ contains both the linear mode frequency, $\omega_{k}$, and a nonlinear shift of the peak of the frequency spectrum, $\delta \omega_{k}$. While previous theories of ion-temperature-gradient-driven turbulence have calculated the linewidth. ${ }^{\circ}$ little attention has been paid to the possibility of a nonlinear shift of the peak of the frequency spectrum. 
While a nonlinear frequency shift is perhaps unlikely to have an impact on $\eta_{i}$ mode stability, it can have a dramatic effect on the particle flux which is sensitive to the real frequency. ${ }^{7}$

The integral over $\omega^{\prime}$ in the renormalized coefficients is analytically calculable using the method of contour integration. By closing the contour in the lower-half plane, only the pole at $\omega_{0}^{\prime}=\omega_{k^{\prime}}^{N L}-i \Delta \omega_{k^{\prime}}$ is enclosed. The integral over $\omega^{\prime}$ constrains the renormalized coefficients to be evaluated at $\omega^{\prime}=\omega_{0}^{\prime}$, i.e.,

$$
\begin{aligned}
& D_{\mathbf{w}}^{x}=\rho_{s}^{2} c_{s}^{2} \sum_{\mathbf{k}^{\prime}} R_{\substack{k-k^{\prime} \\
\omega-\omega_{0}^{\prime}}}^{-1} k_{y}^{\prime 2}\left\langle\left(\frac{e \tilde{\phi}}{T_{e}}\right)^{2}\right\rangle_{\substack{k^{\prime} \\
\omega_{0}^{\prime}}} \\
& \beta_{\underline{k}}^{x}=\rho_{s}^{2} c_{s}^{2} \sum_{\mathbf{k}^{\prime}} R_{\substack{t-k^{\prime} \\
\omega-\omega_{0}^{\prime} \\
-1}} R_{-k_{0}^{\prime}}^{-1} k_{y}^{\prime 2}\left\langle\left(\frac{e \dot{\phi}}{T_{e}}\right)^{2}\right\rangle_{\substack{k^{\prime} \\
\omega_{\mathbf{0}}^{\prime}}}\left\{\omega_{0}^{\prime}-\omega_{* e}^{\prime}\left[1+\eta_{e}\left(\frac{v^{2}}{v_{e}^{2}}-\frac{3}{2}\right)\right]-\beta^{\prime}\right\}
\end{aligned}
$$

Note that when summed over $k_{y}^{\prime}$, most of the real parts of $\beta_{k}^{x}$ and $\beta_{w}^{y}$ will vanish since

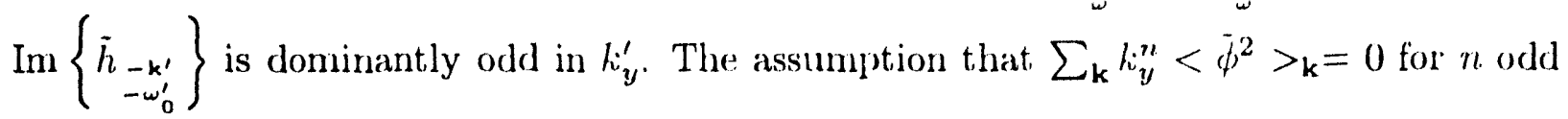
is implicit in this work. If the symmetry of the wavenumber spectrum is broken (e.g., by a sheared radial electric field ${ }^{23}$ ), then this assumption is not valid and the renormalized source terms can be quite different.

In the next section, the renormalized nonlinear coefficients and the frequency spectrum ansatz will be used to calculate the particle flux. The coefficients themselves are evaluated in various limits in Appendix $\mathrm{A}$. 


\section{NONLINEAR PARTICLE FLUX}

In this section, the renormalized trapped electron response and an ansatz of a turbulently broadened frequency spectrum are used to obtain an integral expression for the particle flux. The particle flux is examined both analytically and numerically, including a qualitative discussion of when the nonlinear terms induce inward directed components in the particle flux.

Using the renormalized trapped electron response in Eq. (1), the nonlinear particle flux is given by

$$
\begin{aligned}
& \Gamma_{e}=-n_{e} c_{s} \sum_{\substack{\mathbf{k} \\
\omega}} \rho_{s} k_{y} \operatorname{Im}\left\{\int_{-\infty}^{+\infty} d x \frac{e \tilde{\phi}_{--\mathrm{k}}}{T_{e}}(x) \int d^{3} v F_{\text {max }}(v)\right.
\end{aligned}
$$

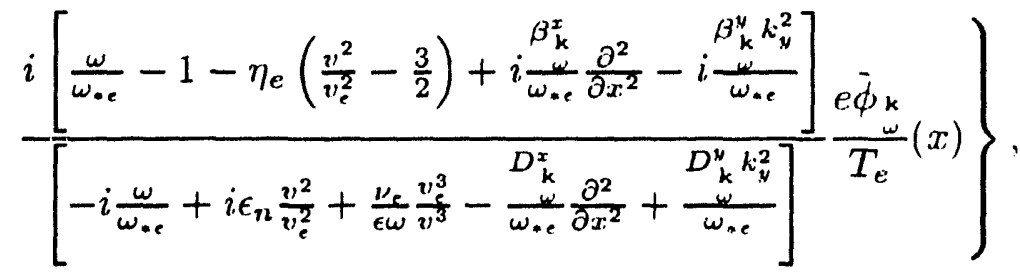

where the integral over the radial coordinate has been expanded from $\pm x_{T}$ to $\pm \infty$. This approximation is valid when the mode amplitude is negligible outside the turning points.

In order to obtain an integral expression for the particle flux, $\bar{\phi}_{\mathbf{k}}(x)$ is expressed in te:ms of the Fourier transformed eigenfunction

$$
\tilde{\phi}_{\boldsymbol{\omega}}(x)=\int d \kappa \exp (-i \kappa x) \tilde{\phi}_{\boldsymbol{\omega}}(\kappa)
$$

Again assuming a Lorentzian frequency spectrum, the particle flux can now be expressed as a sum over $k_{y}$ and an integral over $\kappa, \omega$, and $t=v^{2} / v_{e}^{2}$,

$$
\begin{aligned}
\Gamma_{e}=n_{e} c_{s} \sqrt{\frac{2 \epsilon}{\pi}} \sum_{\mathbf{k}} \rho_{s} k_{y} \int_{-\infty}^{+\infty} d \kappa & \left\langle\left(\frac{e \dot{\phi}}{T_{e}}(k)\right)^{2}\right\rangle_{\mathbf{k}} \int_{0}^{+\infty} d t \sqrt{t} \exp (-t) \int_{-\infty}^{+\infty} d \omega \frac{\Delta \omega_{k} / \pi}{\left(\omega-\omega_{k}^{N L}\right)^{2}+\Delta \omega_{k}^{2}} \\
\times & \operatorname{Im}\left\{\frac{i\left[1-\frac{\omega}{\omega_{e e}}+\eta_{e}\left(t-\frac{3}{2}\right)+\beta_{r}\right]-\beta_{i}}{-i\left[\frac{\omega}{\omega_{e e}}-\epsilon_{n} t-\mu_{i}\right]+\frac{\mu_{e}}{\epsilon \omega_{e c}} t^{-3 / 2}+\mu_{r}}\right\}
\end{aligned}
$$


where $\mu_{r}=\operatorname{Re}\left[\left(D_{\substack{k \\ k}}^{x} \kappa^{2}+D_{k}^{y} k_{y}^{2}\right) / \omega_{* e}\right]$ is the turbulent collisional resonance broadening, $\mu_{i}=\operatorname{Im}\left[\left(D_{k}^{x} \kappa^{2}+D_{k}^{y} k_{y}^{2}\right) / \omega_{* e}\right]$ is the turbulent shift of the collisional resonance peak,

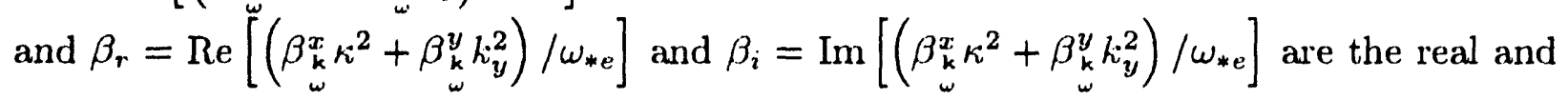
imaginary parts of the renormalized source.

The integral over $\omega$ is once again analytically calculable using the method of contour integration. By closing the contour in the upper-half plane, only the pole at $\omega_{0}=\omega_{k}^{N L}+$ $i \Delta \omega_{k}$ is enclosed. Note that while $\mu_{i}$ and $\beta_{r}$ may be dependent on $\omega$, this does not affect the calculation. The particle flux can now be expressed as

$$
\Gamma_{e}=n_{e} c_{s} \sum_{\mathbf{k}} \int_{-\infty}^{+\infty} d \kappa\left\langle\left(\frac{e \bar{\phi}}{T_{e}}(\kappa)\right)^{2}\right\rangle\left(F_{\nabla n}+F_{\nabla T_{e}}+F_{\beta_{r}}+F_{\beta_{i}}+F_{\Delta \omega_{k}}\right)
$$

where

$$
\begin{aligned}
& F_{\nabla_{n}}=\sqrt{\frac{2 \epsilon}{\pi}} \rho_{s} k_{y} \int_{0}^{\infty} d t \sqrt{t} \exp (-t)\left\{\left(1-\Omega_{k}\right)\left[\left(t_{3} / t\right)^{3 / 2}+\bar{\mu}_{r}+\Delta \Omega_{k}\right] D_{o}^{-1}\right\} \\
& F_{\nabla T_{e}}=\sqrt{\frac{2 \epsilon}{\pi}} \rho_{s} k_{y} \int_{0}^{\infty} d t \sqrt{t} \exp (-t)\left\{\eta_{e}\left(t-\frac{3}{2}\right)\left[\left(t_{3} / t\right)^{3 / 2}+\bar{\mu}_{r}+\Delta \Omega_{k}\right] D_{o}^{-1}\right\},{ }^{\prime} \\
& F_{\beta_{r}}=\sqrt{\frac{2 \epsilon}{\pi}} \rho_{s} k_{y} \int_{0}^{\infty} d t \sqrt{t} \exp (-t)\left\{\bar{\beta}_{r}\left[\left(t_{3} / t\right)^{3 / 2}+\bar{\mu}_{r}+\Delta \Omega_{k}\right] D_{o}^{-1}\right\} \\
& F_{\beta_{1}}=\sqrt{\frac{2 \epsilon}{\pi}} \rho_{s} k_{y} \int_{0}^{\infty} d t \sqrt{t} \exp (-t)\left\{-\bar{\beta}_{i}\left(\Omega_{k}-\epsilon_{n} t-\bar{\mu}_{i}\right) D_{o}^{-1}\right\} \\
& F_{\Delta \omega_{k}}=\sqrt{\frac{2 \epsilon}{\pi}} \rho_{s} k_{y} \int_{0}^{\infty} d t \sqrt{t} \exp (-t)\left\{\Delta \Omega_{k}\left(\Omega_{k}-\epsilon_{n} t-\bar{\mu}_{i}\right) D_{o}^{-1}\right\}
\end{aligned}
$$

and $D_{o}=\left[\Omega_{k}-\epsilon_{n} t-\bar{\mu}_{i}\right]^{2}+\left[\left(t_{3} / t\right)^{3 / 2}+\bar{\mu}_{r}+\Delta \Omega_{k}\right]^{2}$. In Eq. (16) all frequencies have been normalized to the electron drift frequency, $\Omega_{k}=\omega_{k}^{N L} / \omega_{* e^{*}}, \Delta \Omega_{k_{i}}=\Delta \omega_{k_{i}} / \omega_{* k^{*}}$, and $t_{3}=\left(\nu_{e f f} / \omega_{* e}\right)^{2 / 3}$. The overbar on the renormalized coefficients denotes evaluation at $\omega=\omega_{0}$. 
The flux functions have the following physical interpretations. $F_{\nabla_{n}}$ and $F_{\nabla T_{e}}$ represent the quasilinear $\nabla n$ and $\nabla T_{e}$ driven terms, respectively, and are modified by the turbulent broadening of both the frequency spectrum and the electron collisional resonance. $F_{\beta_{r}}$ and $F_{\beta_{i}}$ are terms proportional to the real and imaginary parts of the renormalized source and represent nonlinear access to both the relaxation of the density gradient and offdiagonal driving from the temperature gradient. Finally, $F_{\Delta \omega_{k}}$ is a result of the finite linewidth of the frequency spectrum. Note, however, that the dominant portion of this term, $F_{\Delta \omega_{k}} \sim \Delta \Omega_{k} \Omega_{k} D_{o}^{-1}$, cancels a similar term in $F_{\nabla n}$ and does not contribute to the particle flux.

\section{A. Qualitative Analysis}

By analyzing the flux functions, Eqs. (17a)-(17e), qualitative predictions can be made of when the nonlinear terms will either modify the existing quasilinear pinch terms or produce additional anomalous pinch terms.

1. The sign of the $\nabla n$ driven term, $F_{\nabla n}$, is determined by the difference between the electron diamagnetic frequency and the mode frequency, $i-\omega_{k}^{N L} / \omega_{* e}$. For modes propagating in the ion direction, $\omega_{k}^{N L} / \omega_{* e}<0$, this term is always outward. In order for this term to be inward, there must be a nonlinear shift of the peak of the frequency spectrum such that $\omega_{k}^{N L} / \omega_{* e}>1$. For $\eta_{i}$ modes, this implies the magnitude of the nonlinear frequency shift be greater than the magnitude of the linear frequency, $\mid \omega_{k}^{N L}-$ $\omega_{k}|>| \omega_{k} \mid$. In order to determine if such a shift is possible or to determine the physical origin of such a shift, a self-consistent calculation of the nonlinear effects on the peak of the frequency spectrum is necessary. It is worth noting that recent work on the cross coupling of electron nonlinearities in long wavelength drift wave turbulence has uncovered large finite-amplitude-induced shifts. ${ }^{24.25}$ While it is not, possible to infer from that work alone the existence of such shifts in the present case, such shifts, if 
they exist, would produce a significant inward flux component in high temperature regimes at finite amplitude.

2. The sign of the $\nabla T_{e}$ driven term sign depends on the integral over $\sqrt{t}(t-$ $\left.\frac{3}{2}\right)\left|R_{k}\right|^{2} \operatorname{Im}\left\{R_{k}\right\}$. In the quasilinear analysis the imaginary part of the propagator is given by $\left(t_{3} / t\right)^{3 / 2}$ and the sign of the integral depends on which terms dominate in $\left|R_{k}\right|^{2}$. In the dissipative limit, $\epsilon \omega_{* e} / \nu_{e} \ll 1$, the collision operator dominates the: propagator, $\left|R_{k}\right|^{2} \sim\left(t_{3} / t\right)^{3}$, and the $\nabla T_{e}$ driven term is outward directed. In the collisionless limit $\epsilon \omega_{* e} / \nu_{e} \gg 1$, the frequency dominates the propagator, $\left|R_{k}\right|^{2} \sim\left(\omega / \omega_{* e}\right)^{2}$, and the integral is negative. In the nonlinear particle flux, the additional terms in the propagator have two effects. They tend to squeeze the region in which the dissipative limit is applicable, i.e. the dissipative limit becomes $\nu_{e} / \epsilon \omega_{* e} \gg 1+\mu_{r}+\Delta \omega_{k} / \omega_{* e}$ and in the collisionless limit, the imaginary part of the propagator is dominated by the turbulent collisional resonance broadening and the finite linewidth of the frequency spectrum. $\operatorname{Im}\left\{R_{k}\right\} \sim\left(\mu_{r}+\Delta \Omega_{k}\right)$. Thus, foi strong turbulence in the collisionless limit the $\nabla T_{e}$ driven term is not a strong pinch term. This will be demonstrated more clearly in the numerical analysis.

3. The real part of the renormalized source induces an inward component in the particle flux when $\bar{\beta}_{r}<0$. While this condition is met in several limits (see Appendix A), this term is small for a spectrum symmetric in $k_{y}$, as noted in the previous section.

4. The imaginary part of the renormalized source induces an inward component in the particle flux when $\bar{\beta}_{i}\left(\Omega_{k}-\epsilon_{n} t-\mu_{i}\right)>0$. Again, this condition is generally met as well (Appendix A), but this term is also small for the previously noted reasons.

5. The term $F_{\Delta \omega_{k}}$ is proportional to $\left(\Omega_{k}-\epsilon_{n} t-\mu_{i}\right)$ and thus, for modes propagating in the ion direction, $\Omega_{k}<0$, this term is inward. However, as noted previously, the dominant term $F_{\Delta \omega_{k}} \propto \Delta \Omega_{k} \Omega_{k}$ cancels exactly with a term in $F_{\nabla n}$.

In summation, the nonlinear terms tend to decrease the magnitude of the flux, inhibit. the quasilinear pinch term and produce only weak additional inward directed terms. The 
only exception to this is the case of an extremely large shift of the peak of the frequency spectrum.

\section{B. Numerical Results}

In this section the nonlinear flux functions, Eqs. (17a)-(17e), are numerically integrated over $\mathrm{t}$ for a given $k_{y}$ and $\kappa=1 / \Delta$ through the use of Mathematica. ${ }^{26}$ Two approximations of the nonlinear coefficients have been used. In the first approximation, all recursive dependence on the renormalized coefficients will be ignored. In the second method, an approximate scheme to include the recursive dependence is used. However, since both methods produced the same qualitative results, we will not distinguish them below. Details of the two approximations and the form of the nonlinear coefficients are contained in Appendix B. Due to the interplay of the various nonlinearities, their dependence on fluctuation amplitude, and the fact that the nonlinearities are defined recursively in the strong turbulence limit, quantitative evaluation of the renormalized coefficients is difficult, particularly for strong turbulence. The following analysis is therefore to be interpreted only as a qualitative description of the effect of nonlinear terms on the particle flux.

For comparison, the quasilinear flux function is also evaluated. In the quasilinear analysis, the renormalized coefficients are set to zero as well as the finite linewidth of the frequency spectrum. The nonlinear flux function will be denoted by $F^{N L}=F_{\nabla n}+F_{\nabla T}+$ $F_{\beta_{r}}+F_{\beta_{i}}+F_{\Delta \omega_{k}}$, and the quasilinear flux function by $F^{Q L}=F_{\nabla n}(q)+F_{\nabla T}(q)$. The parameters used are contained in Table 1, except where otherwise noted. The results of both the nonlinear and quasilinear analysis are as follows.

1. Strongly Dissipative Regime.

In Figure 1, the nonlinear flux function is shown as a function of the finite linewidth of the frequency spectrum in the strongly dissipative trapped electron regime, $\epsilon \omega_{* e} / \nu_{\mathrm{e}}=$ 0.1 . The quasilinear flux function is shown for comparison. As noted previously, in this 
regime all terms of the quasilinear flux are outward. ${ }^{7}$ The density and temperature gradient driven components of the flux function are shown in Fig. 1b. The nonlinear $F_{\nabla_{\boldsymbol{n}}}$ and $F_{\nabla T}$ are reduced below their respective quasilinear levels by the finite linewidth. In Fig. 1c, the nonlinear flux terms $F_{\beta_{r}}, F_{\beta_{i}}$, and $F_{\Delta \omega_{k}}$ are shown. While these terms do give rise to inward directed components of the particle flux, they are smaller than the other components of the flux by orders of magnitude due to the cancellations in the renormalized source and the dominantly laminar trapped electron response in this regime.

Figure 2 shows the nonlinear and quasilinear flux functions as a function of the nonlinearly shifted peak of the frequency spectrum in the strongly dissipative regime. The nonlinear shift has been assumed to be in the electron direction, i.e., $\delta \omega_{k}>0$. While the quasilinear flux has been allowed to vary with the peak of the frequency spectrum, it must be nuted that there is some inconsistency in allowing for a finite shift of the peak of the frequency spectrum with zero width, i.e. $\Delta \omega_{k}=0$. It is apparent from Fig. 2b, that the $F_{\nabla n}$ and $F_{\nabla_{n}}(q)$ terms decrease linearly with the shift of the real frequency in this regime. These terms become inwardly directed when the shifted frequency increases above the diamagnetic frequency, $\omega_{k}^{N L}>\omega_{* e}$. While we have denoted this term as the $\nabla n$ driven term, the source of the nonlinear frequency shift has not been specified. Thus, this term may no longer be directly dependent on the density gradient. The quasilinear $\nabla T_{e}$ driven term increases with the increasing frequency, reacting to the electron collisional resonance, $\Omega_{k} \sim \epsilon_{n} t$. In contrast, the nonlinear $\nabla T_{e}$ driven term is insensitive to the collisional resonance due to the finite linewidth of the frequency spectrum.

2. Moderately Dissipative Regime.

In Figures 3 and 4, the nonlinear flux function is shown as a function of the finice linewidth and shifted peak of the frequency spectrum in the moderately dissipative trapped electron regime, $\epsilon \omega_{* e} / \nu_{e}=1.0$. Again, the quasilinear flux function is shown 
for comparison. While the $F_{\nabla n}$ term is again reduced below its quasilinear level by the finite linewidth, the effect on $F_{\nabla T}$ is much more dramatic in this regime (Fig. 2b). The quasilinear $\nabla T_{e}$ component of the particle flux is inward in this regime. While previous analytic estimates of the threshold for this term to become inward have required higher temperatures ${ }^{7}$ (namely, $\epsilon \omega_{* e} / \nu_{e}>1.0$ ), the numerical prediction is lower, $\epsilon \omega_{* e} / \nu_{e} \sim 1$. This is due to the inclusion of the complete propagator over the whole region of integration. The nonlinear $\nabla T_{e}$ component of the flux, however, is outwardly directed (see Fig. $3 b$ ). In this case, $\Delta \omega_{k} / \nu_{e}>1$ and the collisional resonance broadening and finite linewidth dominate the imaginary part of the propagator. Thus, the nonlinear flux is less likely to have an inward directed term in the moderately dissipative regime. The shift of the real frequency also has a strong effect on the quasilinear $\nabla T_{e}$ driven term (Figs. 4a and $4 \mathrm{~b}$ ). This strong effect is due to the shifted frequency approaching the electron collisional resonance, $\omega_{k}^{N L} \sim \omega_{\text {de }}$. The nonlinear flux function is insensitive to this resonance due to the finite linewidth, $\Delta \omega_{k}=\omega_{* e}$. The primary effect of the frequency shift on the nonlinear flux function is once again to induce an a inward component $\left(F_{\nabla n}\right)$ when $\omega_{k}^{N L}>\omega_{* e}$.

3. Weakly Dissipative Regime.

These trends are seen to continue in the weakly dissipative regime, $\epsilon \omega_{* e} / \nu_{e}=10.0$, in Figs. 5 and 6. In Fig. 5a, it is apparent that both the magnitude and sign of the nonlinear flux function are different than the quasilinear flux function. This difference is due primarily to the change in the $F_{\nabla T}$ component (Fig. 5b). While this term is a strong inward pinch in the quasilinear analysis and dominates the particle flux, in the nonlinear analysis this term, while remaining inward, becomes much weaker as $\Delta \omega_{k}$ approaches $\omega_{* e}$. The effect of the shifted frequency (Fig. 6) is the same as in the moderately dissipative case. The quasilinear terms are strongly affected by the collisional resonance, while the nonlinear terms do not even see the resonance. 
The numerical analysis confirms and elucidates many of the points made in the qualitative analysis. Instead of producing new inward components in the particle flux, the nonlinear terms inhibit the quasilinear $\nabla T_{e}$ pinch. 


\section{DISCUSSION}

The nonlinear particle flux for ion-temperature gradient-driven turbulence has been examined in the dissipative trapped electron regime both analytically and numerically. The primary results of this work are as follows.

i. Nonlinear effects can substantially alter the predicted nonlinear particle flux from the quasilinear value.

ii. In the strongly dissipative trapped electron regime, though the electron response remains dominantly laminar, the predicted nonlinear particle flux is still much less than the quasilinear value $\left(\sim \frac{1}{2}\right)$.

iii. In the weakly dissipative or collisionless trapped electron regime, both the sign and magnitude of the nonlinear particle flux can differ from the quasilinear prediction. Specifically, both the finite linewidth of the frequency spectrum and the turbulent broadening of the electron collisional resonance act to inhibit the quasilinear $\nabla T_{e}$ driven inward pinch in this regime. While the total quasilinear particle flux is inward for collisionless trapped electrons, the nonlinear particle flux is outward.

It is interesting to note the recent pellet fueling experiments on the Joint European Torus (JET). ${ }^{2}$ In these experiments, the evolution of the electron density profile was modelled with a particle transport analysis code, PTRANS. ${ }^{27}$ The results of the transport code were compared with predictions of particle transport from theories of $\eta_{i}$-mode turbulence, ${ }^{9,28,20}$ all of which used some form of quasilinear analysis to calculate the particle flux. While the comparison of the quasilinearly predicted and experimentally derived particle diffusivity met with mixed results (there was, however, limited data on ion temperature profiles), there was a marked discrepancy between the strong inward convective velocity predicted by the quasilinear estimates, since the experimental parameters were in the collisionless trapped electron regime, and the experimental results which predicted no anomalous inward flow. It is clear from the results presented in this paper that the 
predicted nonlinear particle flux in the collisionless trapped electron regime has only a very weak inward component, which is in much better agreement with the experimental results than the quasilinear estimates.

This work also presents a cautionary note to those performing numerical simulations of ion temperature gradient driven turbulence. While in the past many of these simulations have assumed adiabatic electrons (i.e., no particle or electron heat transport), there has been some discussion of including the nonadiabatic electron response in order to predict the particle flux. If future numerical simulations consider the collisionless trapped electron regime, then se full nonlinear trapped electron response must be incorporated in order to correctly evaluate the particle flux. 


\section{Acknowledgements}

The authors thank D. E. Newman for useful conversations. This research was supported by the Office of Fusion Energy, U.S. Department of Energy, under Grant DE-FG02-89ER53291.

\section{Appendix A: Renormalized coefficients in various limits}

It is useful to derive expressions for the renormalized coefficients in the dissipative and collisionless limits.

1. Dissipative Regime, $\epsilon \omega_{* e} / \nu_{\mathrm{e}} \ll 1$.

In the dissipative regime, the trapped electron propagator is dominated by the collision

operator, $\frac{\nu_{\epsilon}}{\epsilon} \frac{v_{s}^{3}}{v^{3}}$. Expanding in powers of $\epsilon \omega_{* e} / \nu_{e}$ and assuming $\sum_{\mathbf{k}} k_{y}^{n}\left\langle\bar{\phi}^{2}\right\rangle=0$ for $n$ odd, then to order $\left(\epsilon \omega_{* e} / \nu_{e}\right)^{4}$ the renormalized coefficients are

$$
\begin{aligned}
& \mu_{r}=\alpha_{k}\left(t / t_{3}\right)^{3 / 2}, \\
& \mu_{i}=\alpha_{k}\left(t / t_{3}\right)^{3}\left(\Omega_{k}-\epsilon_{n} t\right), \\
& \beta_{r}=\alpha_{k}\left(t / t_{3}\right)^{9 / 2} \Delta \Omega_{k}\left(\Omega_{k}-\epsilon_{n} t\right), \\
& \beta_{i}=-\alpha_{k}\left(t / t_{3}\right)^{3} \Delta \Omega_{k} .
\end{aligned}
$$

2. Collisionless Regime, $\epsilon \omega_{* e} / \nu_{e} \gg 1$.

In the collisionless regime, the propagator is dominated by the real terms, $\omega-\omega_{d e} \frac{v^{2}}{v_{e}^{2}}$. While in this limit the denominator is more appropriately treated as a delta function. the following are estimates of the sign and magnitude of the coefficients. Expanding in powers of $\left(\epsilon \omega_{* e} / \nu_{e}\right)^{-1}$, the renormalized coefficients are

$$
\begin{aligned}
& \mu_{r}=\left(\alpha_{k} / 2\right)\left(t_{3} / t\right)^{3 / 2}\left(\Omega_{k}-\epsilon_{n} t\right)^{-2}, \\
& \mu_{i}=\left(\alpha_{k} / 2\right)\left(\Omega_{k}-\epsilon_{n} t\right)^{-1}, \\
& \beta_{r}=\left(\alpha_{k} / 4\right)\left\{\Delta \Omega_{k}-2\left(t_{3} / t\right)^{3 / 2}\left[1-\Omega_{k}-\eta_{e}\left(t-\frac{3}{2}\right)\right] / \Omega_{k}\right\}\left(\Omega_{k}-\epsilon_{n} t\right)^{-2},
\end{aligned}
$$




$$
\beta_{i}=-\left(\alpha_{k} / 4\right)\left[1-\Omega_{k}-\eta_{e}\left(t-\frac{3}{2}\right)\right]\left(\Omega_{k}-\epsilon_{n} t\right)^{-2}
$$

3. Strong Turbulence Regime, $\left(\Delta \omega_{k} / \omega_{* e}\right), \mu_{r} \gg\left(\nu_{e} / \epsilon \omega_{* e}\right),\left|\omega / \omega_{* e}\right|$.

In the strong turbulence regime, the propagator is dominated by the collisional resonance broadening and the finite linewidth of the frequency spectrum. With the approximation, $\Delta \omega_{k} / \omega_{* e} \sim \mu_{r}$, the renormalized coefficients are

$$
\begin{aligned}
& \mu_{r}=\sqrt{\alpha_{k} / 2}, \\
& \mu_{i}=\frac{1}{3}\left(\Omega_{k}-\epsilon_{n} t\right), \\
& \beta_{r}=-\left(\alpha_{k} / 3\right)\left(\Omega_{k}-\epsilon_{n} t\right)\left(\mu_{r}+\Delta \Omega_{k}\right)^{-2}, \\
& \beta_{i}=-\left(\alpha_{k} / 3\right)\left(\mu_{r}+\Delta \Omega_{k_{k}}\right)^{-1} .
\end{aligned}
$$

While the above approximations must be taken as rough estimates, they can be used to make the following observations. $\mu_{r}>0$ and $\mu_{2}<0$ (for modes propagating in the ion direction) in all of the above cases. Both $\beta_{r}$ and $\beta_{i}$ are generally less than zero for modes propagating in the ion direction.

\section{Appendix B: Approximations for Numerical Analysis}

In the simplest approximation of the nonlinear coefficients, all recursive dependence is ignored. While care must be taken to determine which terms vanish when summed over $\mathbf{k}^{\prime}$, subsequently we do not differentiate between $\Omega_{k}$ and $\Omega_{k^{\prime}}$, etc. The coefficients used for numerical integration are then given by

$$
\begin{aligned}
\bar{\mu}_{r} & =\alpha_{k}\left(t / t_{3}\right)^{3 / 2} A_{1} A_{2} N_{0}^{-1} \\
\bar{\mu}_{i} & =\alpha_{k}\left(t / t_{3}\right)^{3} B_{1} A_{2}^{2} N_{0}^{-1}, \\
\bar{\beta}_{r} & =\alpha_{k}\left(t / t_{3}\right)^{0 / 2} B_{1}\left\{\Delta \Omega_{k}\left[A_{3} A_{2}^{2}-2\left(t / t_{3}\right)^{3} A_{2} B_{1}^{2}\right)\right. \\
& \left.\quad-\left(t / t_{3}\right)^{3 / 2} B_{1} B_{2}\left(A_{2}^{2}+2 A_{2} A_{3}\right)\right\} N_{1}^{-1} N_{0}^{-1} .
\end{aligned}
$$




$$
\begin{aligned}
\bar{\beta}_{i}=\alpha_{k}\left(t / t_{3}\right)^{3}\left\{\Delta \Omega_{k}\left[A_{1} A_{2} A_{3}-\left(t / t_{3}\right)^{3} A_{2}^{2} B_{1}^{2}\right]\right. \\
\left.-\left(t / t_{3}\right)^{3 / 2} B_{1} B_{2}\left(A_{3} A_{2}^{2}+A_{1} A_{2}\right)\right\} N_{1}^{-1} N_{0}^{-1},
\end{aligned}
$$

where $A_{1}=1+4 \Delta \Omega_{k}\left(t / t_{3}\right)^{3 / 2}+2\left(t / t_{3}\right)^{3}\left[2 \Delta \Omega_{k}^{2}+\left(\Omega_{k}-\epsilon_{n} t^{2}\right)^{2}\right], A_{2}=1+2 \Delta \Omega_{k}\left(t / t_{3}\right)^{3 / 2}$, $A_{3}=1+\Delta \Omega_{k}\left(t / t_{3}\right)^{3 / 2}, B_{1}=\Omega_{k}-\epsilon_{n} t, B_{2}=1-\Omega_{k}+\eta_{e}\left(t-\frac{3}{2}\right), N_{0}=A_{1}^{2}+4\left(t / t_{3}\right)^{3} B_{1}^{2}$ and $N_{1}=A_{3}^{2}+\left(t / t_{3}\right)^{3} B_{1}^{2}$.

In the second method of approximating the nonlinear coefficients, a two-step method is used in order to capture some properties of the recursive definitions appropriate for the strong turbulence limit. First, with the approximation $\Omega_{k}=\Omega_{k^{\prime}}$ (after cancellations of odd powers of $\mathbf{k}^{\prime}$ ).

$$
\bar{\mu}_{r}\left[\bar{\mu}_{r}+2 \Delta \Omega_{k}+\left(t_{3} / t\right)^{3 / 2}\right]=\alpha_{k}\left\{1-\frac{2 B_{1}^{2}}{4 B_{1}^{4}+\left[\bar{\mu}_{r}+2 \Delta \Omega_{k}+\left(t_{3} / t\right)^{3 / 2}\right]^{2}}\right\} .
$$

The second term in the curly brackets on the righthand side of Eq. (34) is less than 1/2. We thus recursively define $\bar{\mu}_{r}$ as

$$
\bar{\mu}_{r}=\frac{1}{2}\left[2 \Delta \Omega_{k}+\left(t_{3} / t\right)^{3 / 2}\right]\left\{\left(1+\frac{4 \alpha_{k} C_{0}}{\left[2 \Delta \Omega_{k}+\left(t_{3} / t\right)^{3 / 2}\right]^{2}}\right)^{\frac{1}{2}}-1\right\} .
$$

where

$$
\begin{aligned}
& C_{0}=1-\left[2+\left[\bar{\mu}_{r \cdot 0}+2 \Delta \Omega_{k}+\left(t_{3} / t\right)^{3 / 2}\right]^{2} / 2\left[B_{1}-\bar{\mu}_{i 0}\right]^{2}\right]^{-1}, \\
& \bar{\mu}_{r \cdot 0}=\frac{1}{2}\left[2 \Delta \Omega_{k}+\left(t_{3} / t\right)^{3 / 2}\right]\left\{\left(1+\frac{4 \alpha_{k}}{\left[2 \Delta \Omega_{k}+\left(t_{3} / t\right)^{3 / 2}\right]^{2}}\right)^{\frac{1}{2}}-1\right\} .
\end{aligned}
$$

The shift of the collisional resonance has been similarly recursively defined. Using $\bar{H}_{i l}=$ $\alpha_{k} B_{1} /\left[4 B_{1}^{2}+\left(\bar{\mu}_{r 0}+2 \Delta \Omega_{k}+\left(t_{3} / t\right)^{3 / 2}\right)^{2}\right], \bar{\mu}_{i}$ is defined as

$$
\bar{\mu}_{i}=\alpha_{k} B_{1} /\left[4\left(B_{1}-\bar{\mu}_{i 0}\right)^{2}+\left(\bar{\mu}_{r}+2 \Delta \Omega_{k}+\left(t_{3} / t\right)^{3 / 2}\right)^{2}\right] .
$$


The renormalized source terms have also been approximated in this manner. By assuming $\beta \sim \beta^{\prime}$, the following forms were derived for numerical integration,

$$
\begin{aligned}
& \bar{\beta}_{r}=-2 \alpha_{k} B_{3}^{2} N_{3} N_{4}^{-1} \frac{\left\{\left[1-\alpha_{k} N_{3} N_{5} N_{4}^{-1}\left(1+N_{6} N_{7}\right)\right] B_{2}+\Delta \Omega_{k}\left(\Delta \Omega_{k}+2 A_{4}\right)\right\}}{\left[\left(1+2 \alpha_{k} B_{3}^{2} N_{3}\right)\left(1-\alpha_{k} N_{3} N_{5} N_{4}^{-1}\right)-2 \alpha_{k}^{2} B_{3}^{2} N_{3}^{2} N_{4}^{-2} N_{5} N_{6} N_{7}\right]}, \\
& \bar{\beta}_{i}=-\alpha_{k} N_{3} N_{5} N_{4}^{-1} \frac{\left\{\left[1+2 \alpha_{k} B_{3}^{2} N_{3} N_{4}^{-1}\left(1+N_{6} N_{7}\right)\right] \Delta \Omega_{k}+N_{6} B_{2}\right\}}{\left[\left(1+2 \alpha_{k} B_{3}^{2} N_{3}\right)\left(1-\alpha_{k} N_{3} N_{5} N_{4}^{-1}\right)-2 \alpha_{k}^{2} B_{3}^{2} N_{3}^{2} N_{4}^{-2} N_{5} N_{6} N_{7}\right]}
\end{aligned}
$$

where $B_{3}=B_{1}-\bar{\mu}_{i}, N_{3}=A_{4} A_{5}-B_{3}^{2}, N_{4}=\left(A_{4}^{2}+B_{3}^{2}\right) A_{5}^{2} N_{5}, N_{5}=A_{5}^{2}+2 B_{3}^{2}, N_{6}=$ $B_{3}\left(2 A_{4} A_{5}^{2}+\Delta \Omega_{k} N_{5}\right) N_{3}^{-1} N_{5}^{-1}, N_{7}=\left(2 A_{4}+\Delta \Omega_{k}\right) N_{3}^{-1}, A_{4}=\left(t_{3} / t\right)^{3 / 2}+\bar{\mu}_{r}+\Delta \Omega_{k}$, and $A_{5}=A_{4}+\Delta \Omega_{k}$.

Finally, as stated previously, in order to calculate the quasilinear particle flux the nonlinear coefficients were set to zero $\left(\alpha_{k}=0\right)$ as well as the finite linewidth, $\Delta \Omega_{k}=0$. 


\section{References}

1 S. M. Wolfe and M. Greenwald, Nucl. Fusion 26, 329 (1986).

2 L. R. Baylor, W. A. Houlberg, S. L. Milora, G. L. Schmidt, and P. Kupschus, Nucl. Fusion 31, 1249 (1991).

3 J. Luxon, R. Anderson, F. Batty, C. Baxi, G. Bramson, N. Brooks, B. Brown, B. Burley, K. H. Burrell, R. Callis, G. Campbell, T. Carlstrom, A. Colleraine, J. Cummings, L. Davis, J. DeBoo, S. Ejima, R. Evanko, H. Fukumoto, R. Gallix, J. Gilleland, T. Glad, P. Gohil, A. Gootgeld, R. J. Groebner, S. Hanai, J. Haskovec, E. Heckman, M. Heiberger, FJH, N. Hosogane, C. Hsieh, G. L. Jackson, G. Jahns, G. Janeschitz, E. Johnson, A. Kellman, J. S. Kim, J. Kohli, A. Langhorn, L. Lao, P. Lee, S. Lightner, J. Lohr, M. Mahdavi, M. Mayberry, B. McHarg, T. McKelvey, R. Miller, C. P. Moeller, D. Moore, A. Nerem, P. Noll, T. Ohkawa, N. Ohyabu, T. Osborne, D. Overskei, P. Petersen, T. Petrie, J. Phillips, R. Prater, J. Rawls, E. Reis, D. Remsen, P. Riedy, P. Rock, K. Schaubel, D. Schissel, J. Scoville, R. Seraydarian, M. Shimada, T. Shoji, B. Sleaford, J. Smith, Jr., P. Smith, T. Smith, R. T. Snider, R. D. Stambaugh, R. Stav, H. St. John, R. Stockdale, E. J. Strait, R. Street, T. S. Taylor, J. Tooker, M. Tupper, S. K. Wong, and S. Yamaguchi, Plasma Physics and Controlled Nuclear Fusion Research (IAEA, Vienna, 1987), Vol. I, p. 159.

4 P. C. Efthimion, D. K. Mansfield, B. C. Stratton, E. Synakowski, A. Bhattacharjee, H. Biglari, P. H. Diamond, R. J. Goldston, C. C. Hegna, D. McCune, G. Rewoldt, S. Scott, W. M. Tang, G. Taylor, R. E. Waltz, R. M. Wieland, and M. C. Zarnstorff, Phys. Rev. Lett. 66, 421 (1991).

5 K. W. Gentle, B. Richards, and F. Waelbroek, Plasma Phys. Controlled Fusion 29. 1077 (1987).

6 R. V. Bravenec, D. W. Ross, P. M. Schoch, D. L. Brower, J. W. Heard, R. L. Hickok, P. W. Terry, A. J. Wootton, and Xuanzong Yang, Nucl. Fusion 31, 687 (1991). 
7 P. W. Terry, Phys. Fluids B 1, 1932 (1989).

8 B. Coppi and C. Spight, Phys. Rev. Lett. 41, 551 (1978).

9 G. S. Lee and P. H. Diamond, Phys. Fluids 29, 3291 (1986).

10 R. E. Waltz and R. R. Dominguez, Phys. Fluids B 1, 1935 (1989).

11 A. S. Ware, P. H. Diamond, H. Biglari, B. A. Carreras, L. A. Charlton, J.-N. Leboeuf, and A. J. Wootton, Phys. Fluids B 4, 877 (1992).

12 F. Y. Gang, P. H. Diamond, J. A. Crotinger, and A. E. Koniges, Phys, Fluids B 3, $955(1991)$.

13 K. C. Shaing and R. D. Hazeltine, Phys. Fluids B 2, 2353 (1990).

14 T. H. Dupree and D. J. Tetrault, Phys. Fluids 21, 425 (1978).

15 P. H. Diamond, P. L. Similon, P. W. Terry, C. W. Horton, S. M. Mahajan, J. D. Meiss, M. N. Rosenbluth, K. Swartz, T. Tajima, R. D. Hazeltine, and D. W. Ross, Plasma Physics and Controlled Nuclear Fusion Research (IAEA, Vienna, 1982), Vol. I, p. 259; P. W. Terry and P. H. Diamond, in Statistichal Physics and Chaos in Fusion Plasmas, ed. by W. Horton and I. Reichl (Wiley, New York, 1984) p. 335.

16 P. W. Terry and W. Horton, Phys. Fluids 26, 169 (1983).

17 N. Mattor and P. W. Terry, Phys. Fluids B 4, 1126 (1992).

18 L. Chen, R. L. Berger, J. G. Lominadze, M. N. Rosenbluth, and P. H. Rutherford, Phys. Rev. Lett. 39, 754 (1977)

10 N. Mattor and P. H. Diamond, Phys. Fluids 31, 1180 (1988).

20 P. W. Terry, J.-N. Leboeuf, P. H. Diamond, D. R. Thayer, J. E. Sedlak, and G. S. Lee, Phys. Fluids 31, 2920 (1988).

21 F. Y. Gang, P. H. Diamond, and M. N. Rosenbluth, Phys. Fluids B 3, 68 (1991).

22 P. W. Terry and P. H. Diamond, Phys. Fluids 28, 1419 (1985).

23 B. A. Carreras, K. Sidikman, P. H. Diamond, P. W. Terry, and L. Garcia, Phys. Fluids B 4, 3115 (1993). 
24 Y.-M. Liang, P. H. Diamond, X.-H. Wang, D. E. Newman, and P. W. Terry, Phys. Fluids B 5, 1128 (1993).

25 D. E. Newman, P. W. Terry, P. H. Diamond, and Y.-M. Liang, Phys. Fluids B 5, 1140 (1993).

20 Wolfram Research, Mathematica (Wolfram Research, Inc., Champaign, Illinois, 1992), Version 2.0.

27 L. R. Baylor, Particle transport in pellet-fueled JET plasmas, PhD Thesis, Univ. of Tennessee, Knoxville (1989)

28 H. Biglari, P. H. Diamond, and M. N. Rosenbluth, Phys. Fluids B 1, 109 (1989)

29 F. Romanelli and S. Briguglio, Phys. Fluids B 2, 754 (1990) 
TABLE I. Equilibrium Parameters

\begin{tabular}{llll}
\hline$\epsilon$ & 0.15 & $\epsilon_{n}$ & 0.1 \\
$\eta_{e}$ & 2.0 & $\alpha_{k}$ & 1.0 \\
$k_{y} \rho_{s}$ & 0.1 & $\kappa \rho_{s}$ & 0.1 \\
$\omega_{k}^{N L} / \omega_{* e}$ & -0.5 & $\Delta \omega_{k} / \omega_{* e}$ & 1.0 \\
\hline
\end{tabular}




\section{Figure Captions}

Fig. 1. The nonlinear flux function as a function of the finite linewidth of the frequency spectrum in the dissiaptive regime, $\epsilon \omega_{* e} / \nu_{e}=0.1$. The quasilinear flux function is shown for comparison. a)The total nonlinear $\left(F^{N L}\right)$ and quasilinear $\left(F^{Q L}\right)$ flux functions, b)the $\nabla n$ and $\nabla T_{e}$ components, and c)the nonlinearly driven components.

Fig. 2. The nonlinear and quasilinear flux functions a function of the nonlinearly shifted peak of the frequency spectrum in the dissiaptive regime, $\epsilon \omega_{* e} / \nu_{e}=0.1$. a)The total nonlinear $\left(F^{N L}\right)$ and quasilinear $\left(F^{Q L}\right)$ flux functions, b)the $\nabla n$ and $\nabla T_{e}$ components, and c)the nonlinearly driven components.

Fig. 3. The nonlinear flux function as a function of the finite linewidth of the frequency spectrum in the moderately dissiaptive regime, $\epsilon \omega_{* e} / \nu_{e}=1.0$. The quasilinear flux function is shown for comparison. a)The total nonlinear $\left(F^{N L}\right)$ and quasilinear $\left(F^{Q L}\right)$ flux functions, b)the $\nabla n$ and $\nabla T_{e}$ components, and c)the nonlinearly driven components.

Fig. 4. The nonlinear and quasilinear flux functions a function of the nonlinearly shifted peak of the frequency spectrum in the moderately dissiaptive regime, $\epsilon \omega_{* e} / \nu_{e}=$ 1.0. a) The total nonlinear $\left(F^{N L}\right)$ and quasilinear $\left(F^{Q L}\right)$ flux functions, b)the $\nabla n$ and $\nabla T_{e}$ components, and c)the nonlinearly driven components.

Fig. 5. The nonlinear flux function as a function of the finite linewidth of the frequency spectrum in the weakly dissiaptive regime, $\epsilon \omega_{* e} / \nu_{\varepsilon}=10.0$. The quasilinear flux function is shown for comparison. a)The total nonlinear $\left(F^{N L}\right)$ and quasilinear $\left(F^{Q L}\right)$ flux functions, b)the $\nabla n$ and $\nabla T_{e}$ components, and c)the nonlinearly driven components.

Fig. 6. The nonlinear and quasilinear flux functions a function of the nonlinearly shifted peak of the frequency spectrum in the weakly dissiaptive regime, $\epsilon \omega_{* e} / \nu_{e}=10.0$. 
a) The total nonlinear $\left(F^{N L}\right)$ and quasilinear $\left(F^{Q L}\right)$ flux functions, b)the $\nabla n$ and $\nabla T_{e}$ components, and c)the nonlinearly driven components.

\section{DISCLAIMER}

This report was prepared as an account of work sponsored by an agency of the United States Government. Neither the United States Government nor any agency thereof, nor any of their employees, makes any warranty, express or implied, or assumes any legal liability or responsibility for the accuracy, completeness, or usefulness of any information, apparatus, product, or process disclosed, or represents that its use would not infringe privately owned rights. Reference herein to any specific commercial product, process, or service by trade name, trademark, manufacturer, or otherwise does not necessarily constitute or imply its endorsement, recommendation, or favoring by the United States Government or any agency thereof. The views and opinions of authors expressed herein do not necessarily state or reflect those of the United States Government or any agency thereof. 

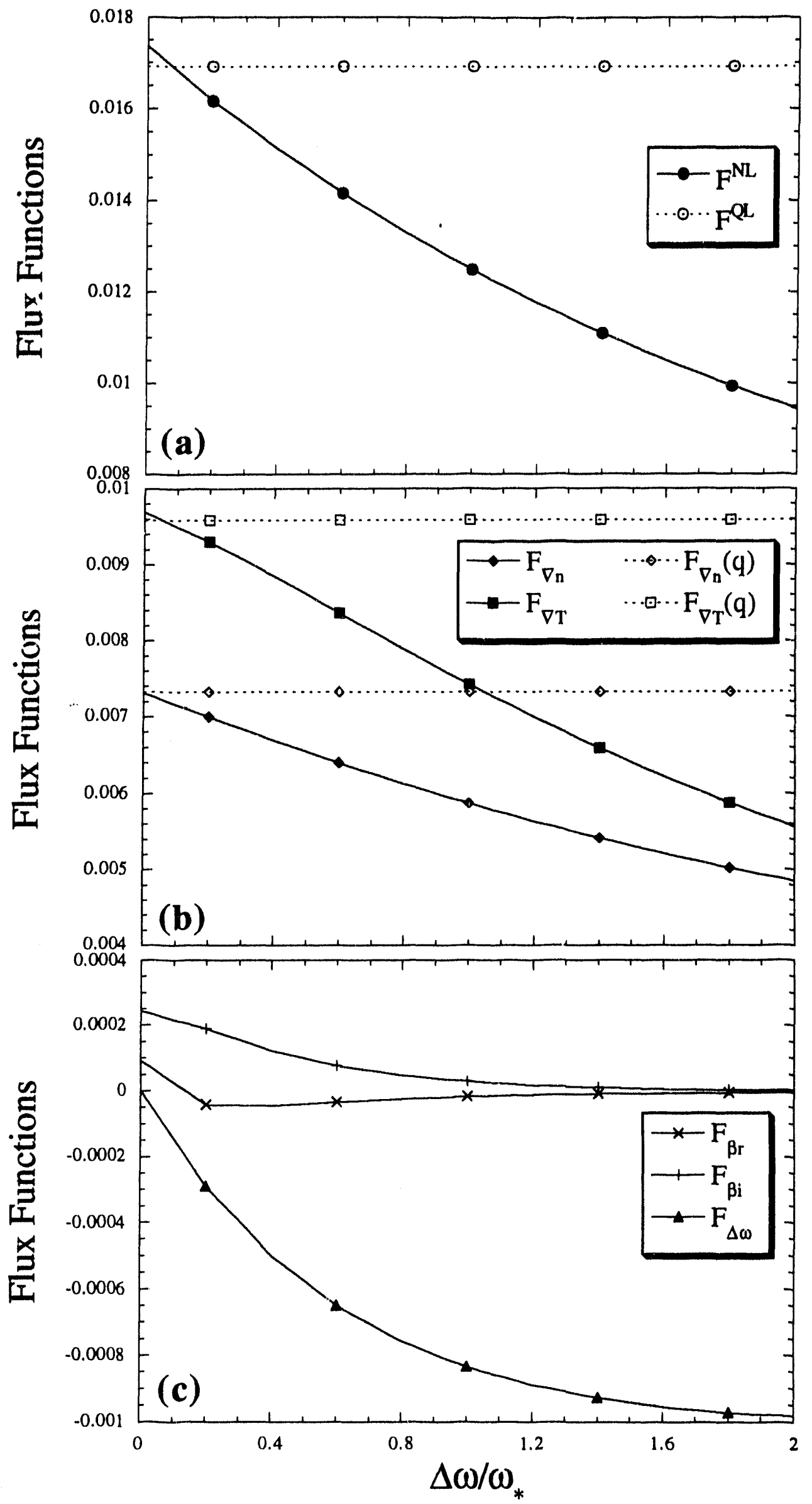

Figure 1 


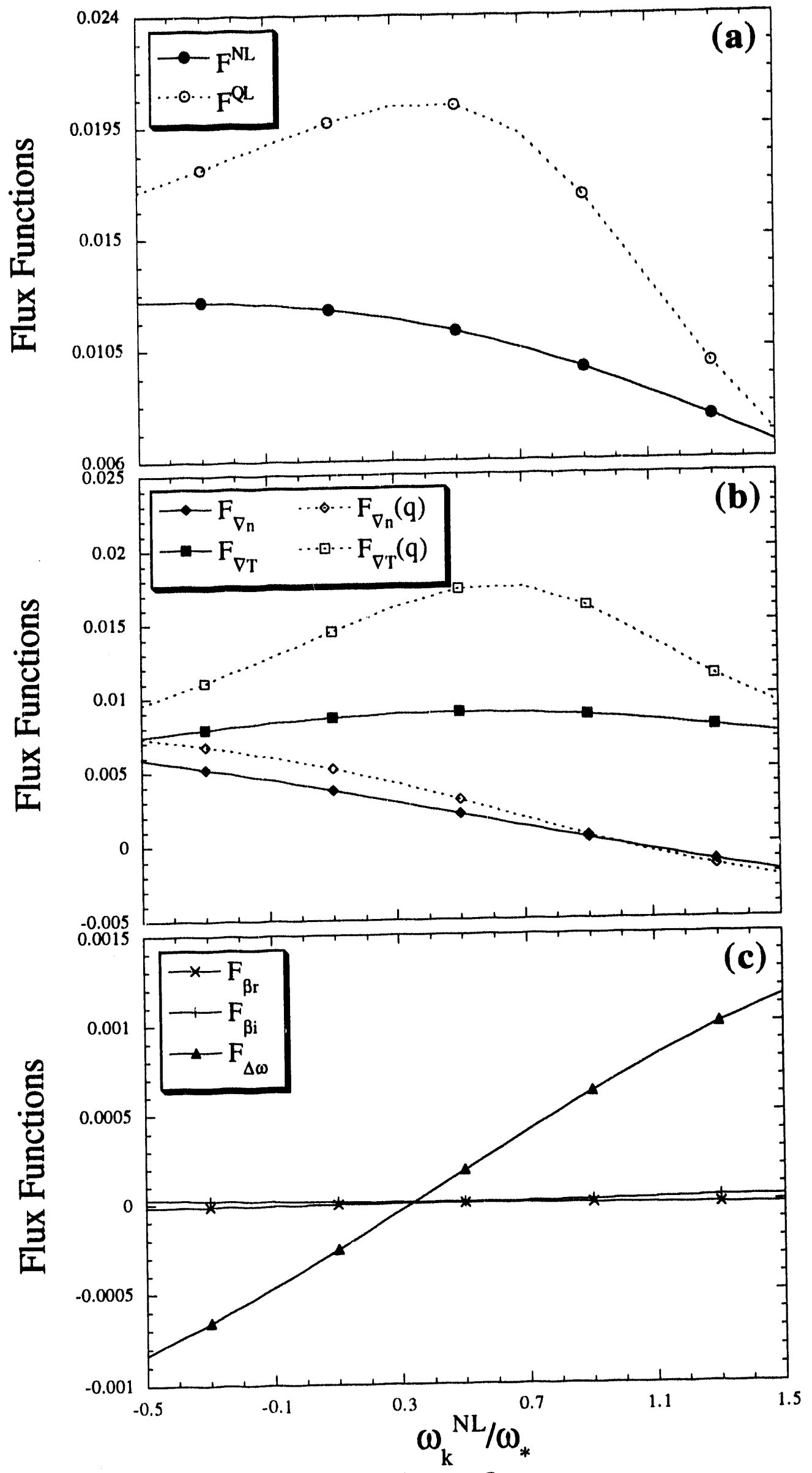

Figure 2 


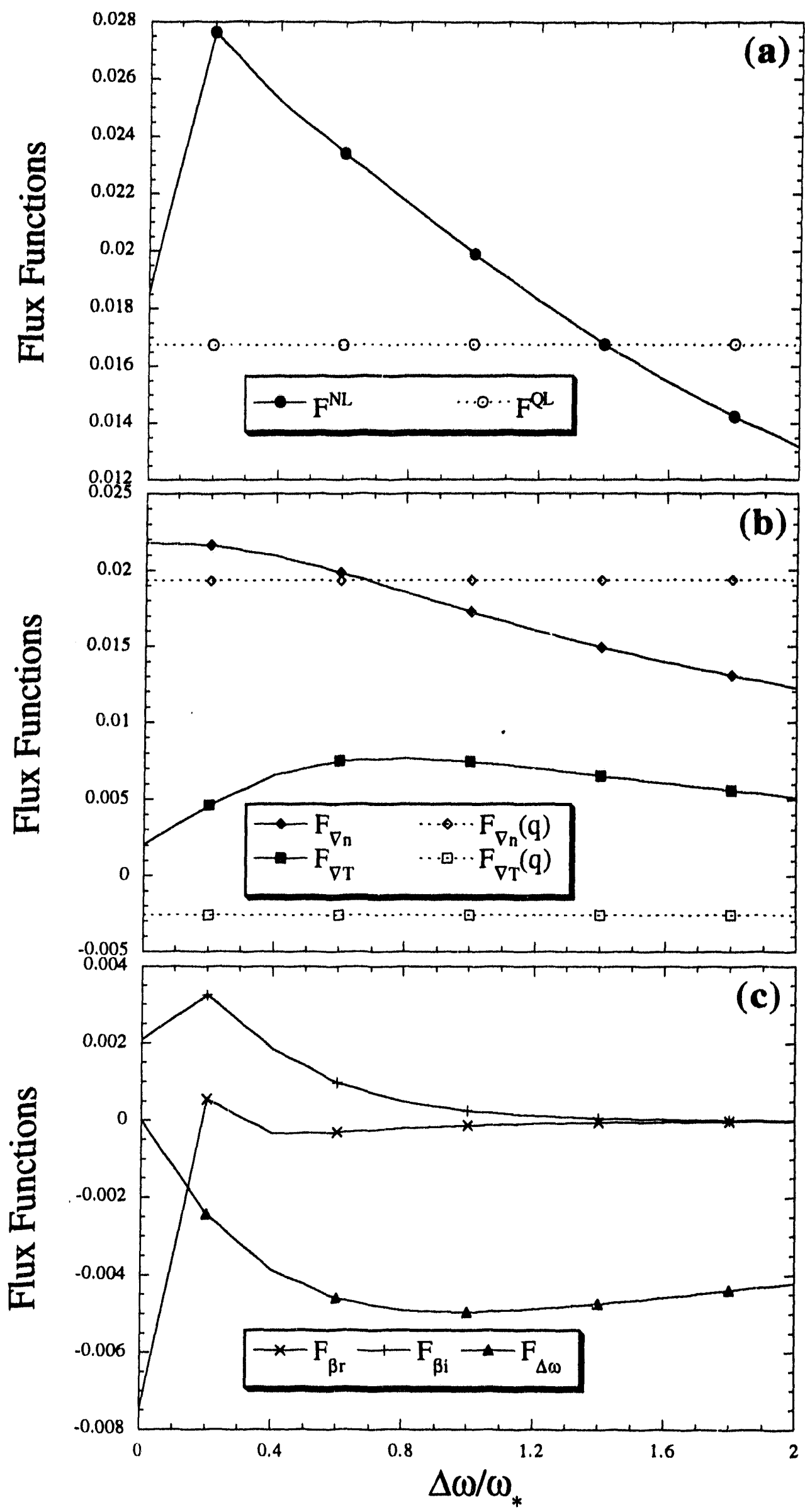

Figure 3 

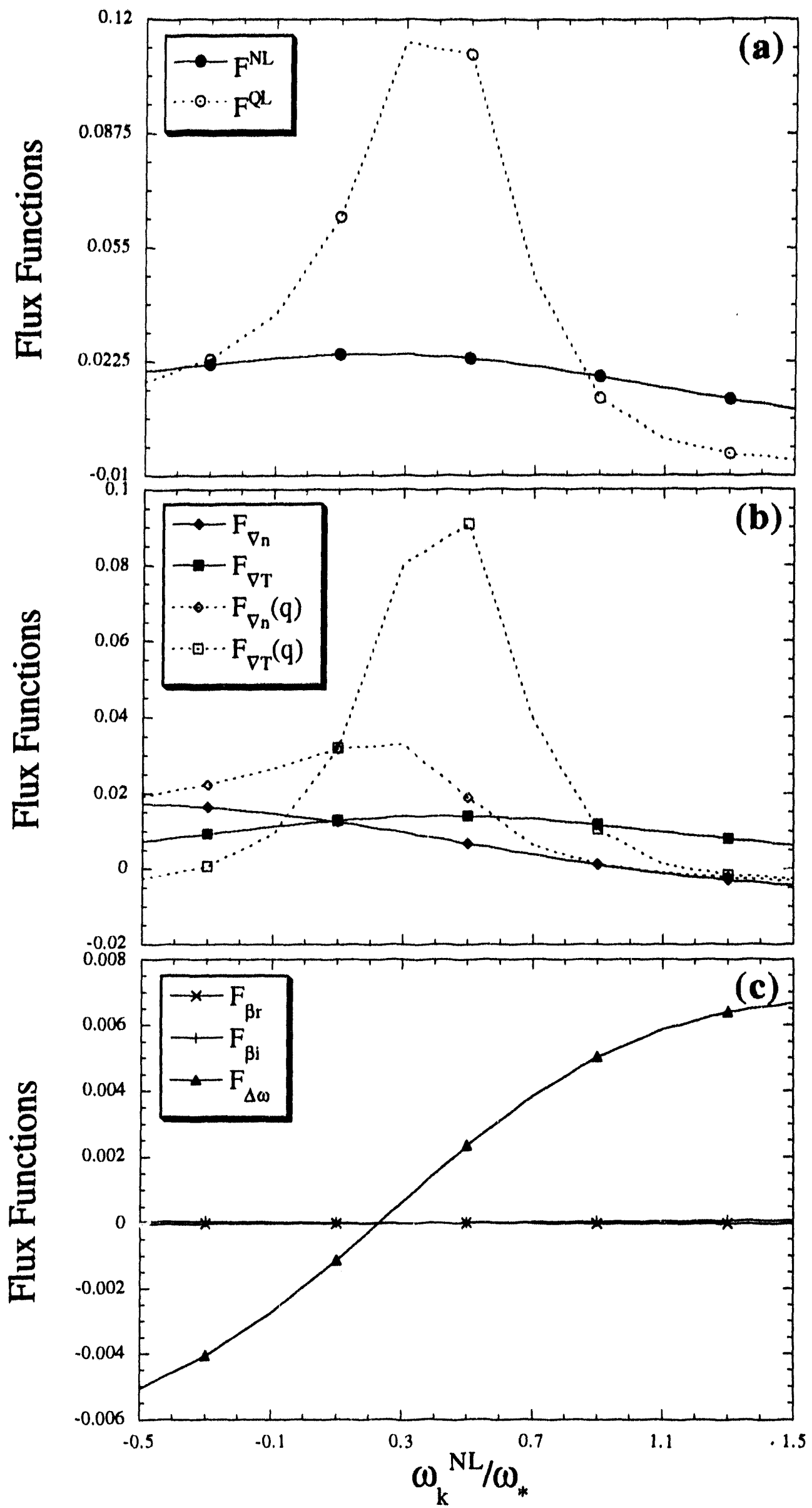

Figure 4 


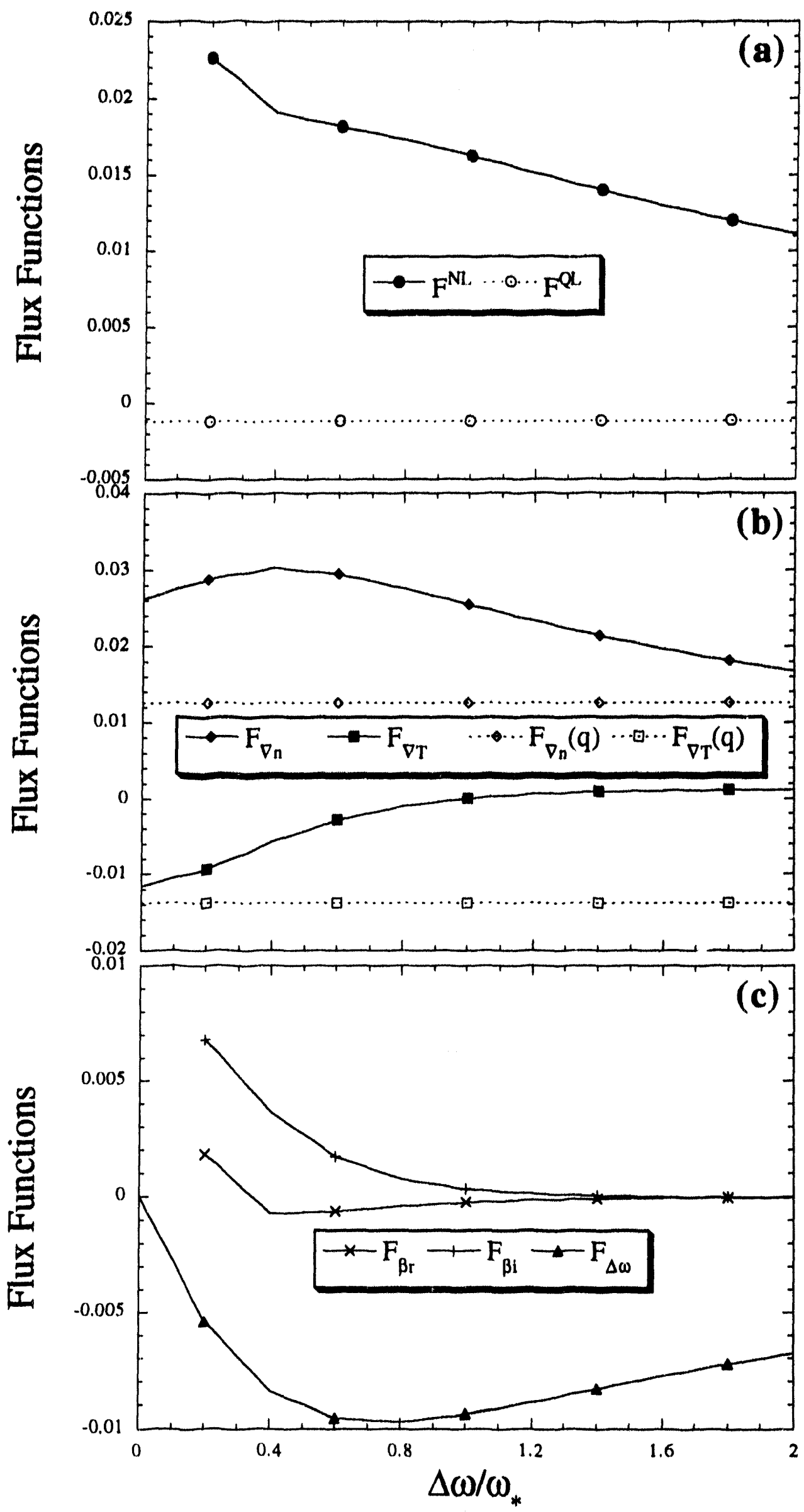

Figure 5 

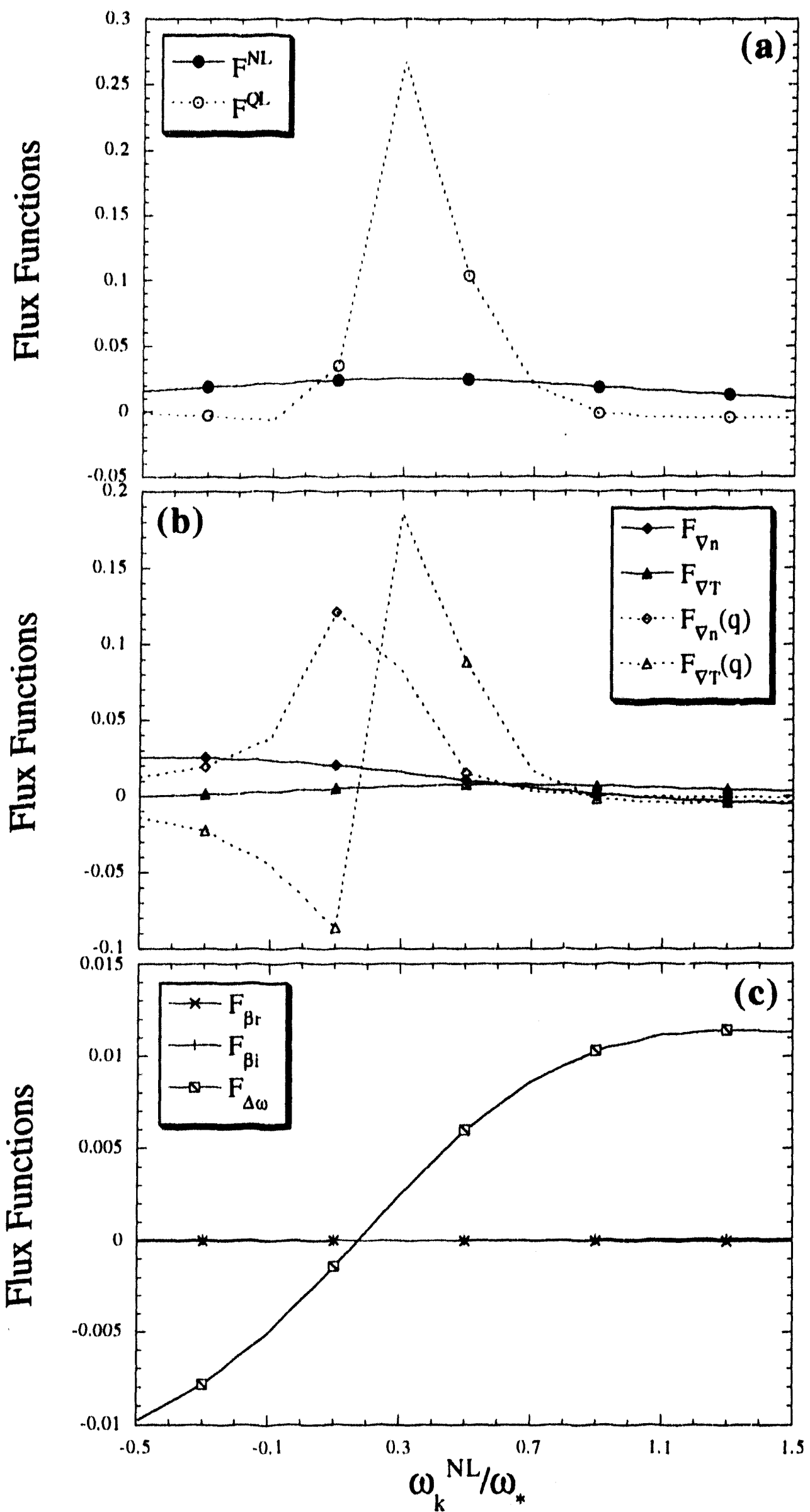

Figure 6 

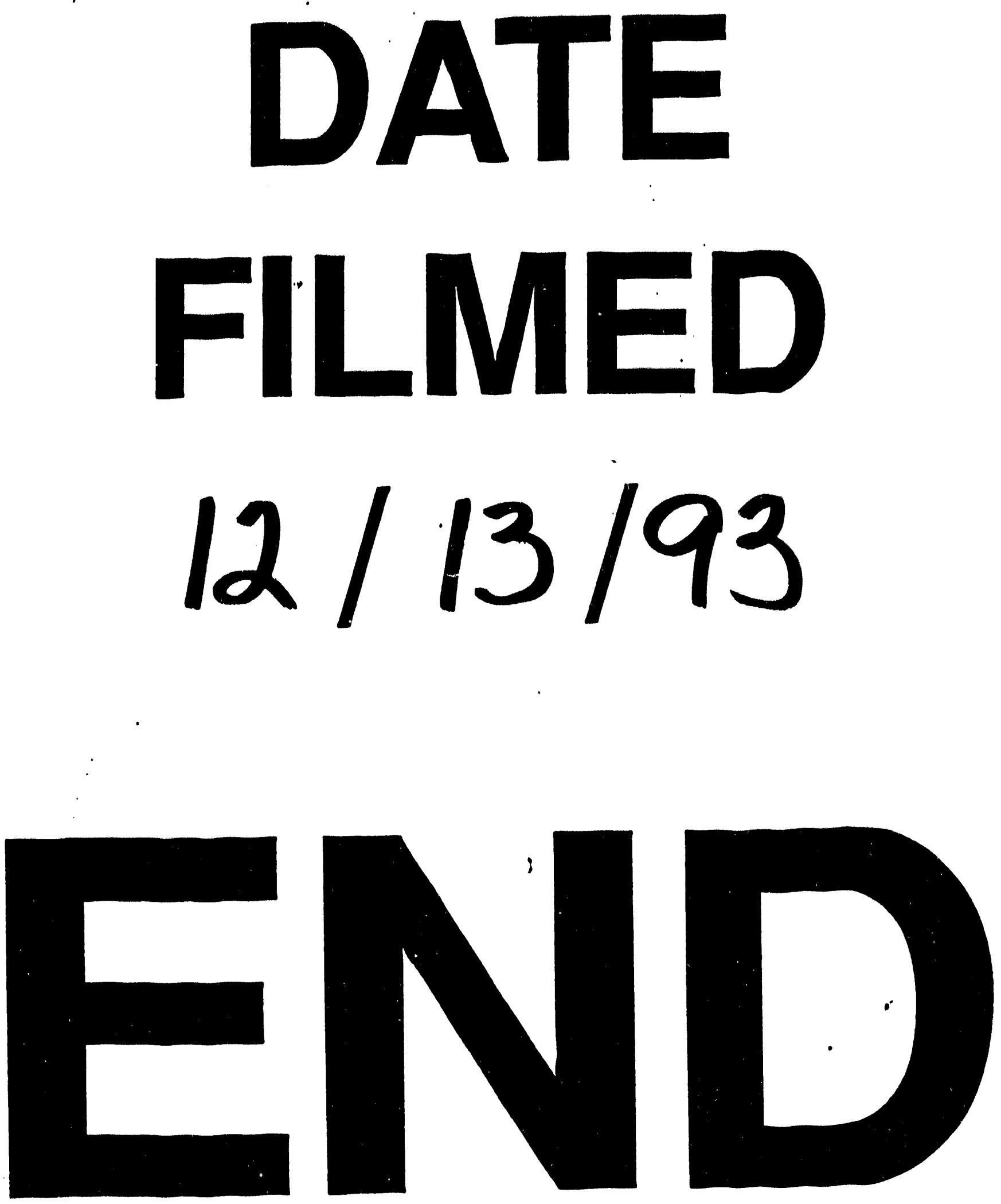
$$
\text { - _ - }
$$

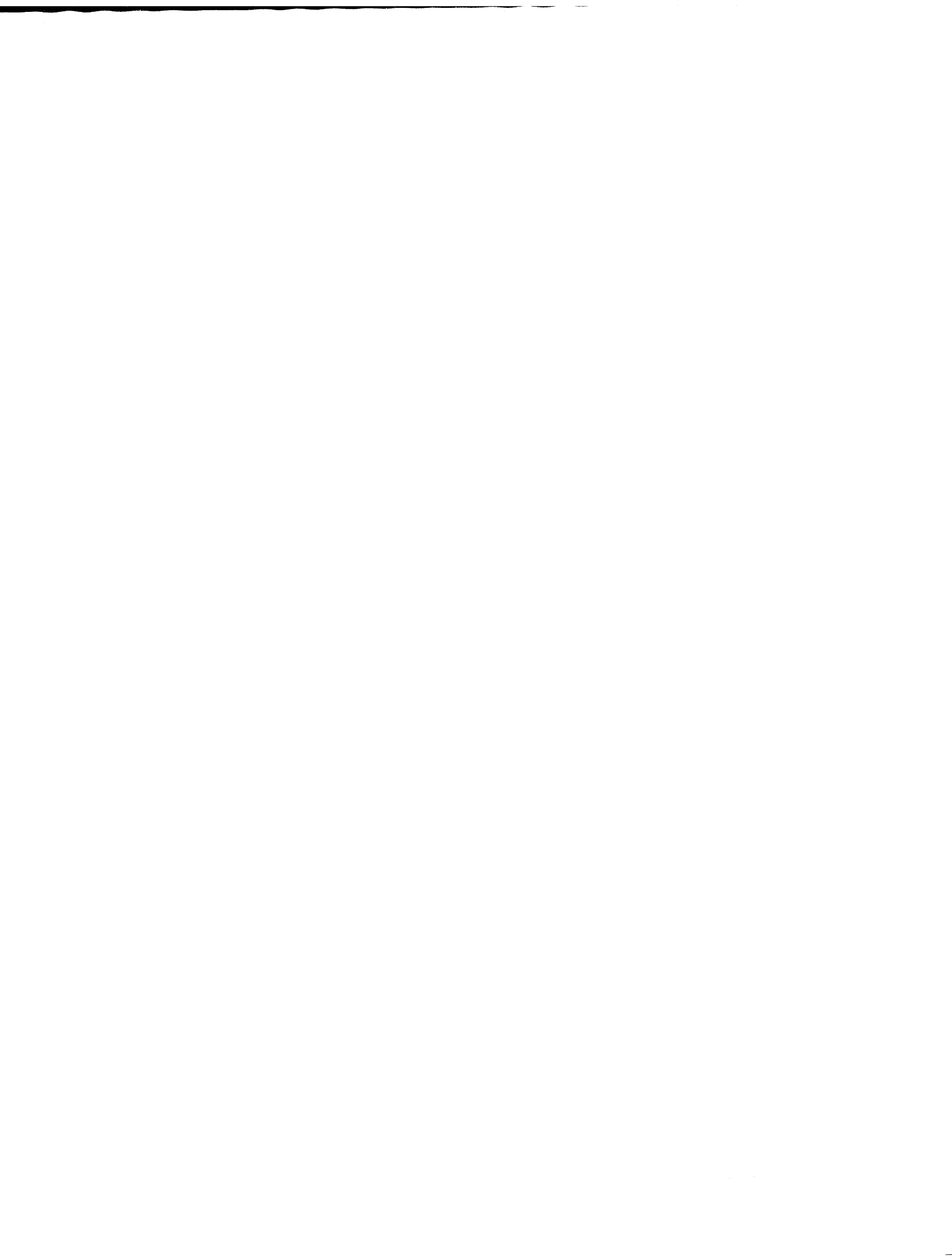

